\title{
GEOMETRY AND HOLONOMY OF INDECOMPOSABLE CONES
}

\author{
DMITRI ALEKSEEVSKY, VICENTE CORTÉS, AND THOMAS LEISTNER
}

\begin{abstract}
We study the geometry and holonomy of semi-Riemannian, time-like metric cones that are indecomposable, i.e., which do not admit a local decomposition into a semiRiemannian product. This includes irreducible cones, for which the holonomy can be classified, as well as non irreducible cones. The latter admit a parallel distribution of null $k$-planes, and we study the cases $k=1$ and $k=2$ in detail. In these cases, i.e., when the cone admits a distribution of parallel null tangent lines or planes, we give structure theorems about the base manifold. Moreover, in the case $k=1$ and when the base manifold is Lorentzian, we derive a description of the cone holonomy. This result is obtained by a computation of certain cocycles of indecomposable subalgebras in $\mathfrak{s o}(1, n-1)$.
\end{abstract}

\section{Contents}

1. Introduction

2. Preliminaries

3. Cones with parallel null lines

4. Metrics of the form $\widetilde{g}=2 \mathrm{~d} u \mathrm{~d} v+u^{2} g$

5. Results about indecomposable subalgebras of $\mathfrak{s o}(t+1, s+1)$

6. Holonomy of metrics $\widetilde{g}=2 \mathrm{~d} u \mathrm{~d} v+u^{2} g$

7. Cones with parallel null 2-planes

References

\section{INTRODUCTION}

1.1. Background. Cone constructions are a valuable tool in differential geometry to study overdetermined PDEs on manifolds. They are applied in conformal [13, 14] and projective geometry [29, 3], but the most striking example is Bär's classification of Riemannian manifolds with real Killing spinors [4]. Bär's observation that real Killing spinors on a Riemannian manifold $(M, g)$ correspond to parallel spinors on the cone

$$
\left(\widetilde{M}=\mathbb{R}^{>0} \times M, \check{g}=d r^{2}+r^{2} g\right),
$$

2010 Mathematics Subject Classification. Primary 53C50; Secondary 53C29, 53B30.

Key words and phrases. Lorentzian manifolds, pseudo-Riemannian manifolds, metric cones, special holonomy.

This work was supported by the Australian Research Council via the grants FT110100429 and DP120104582 and by the German Science Foundation (DFG) under the Research Training Group 1670 and under Germany's Excellence Strategy - EXC 2121 "Quantum Universe" - 390833306. D.A. is supported by grant n 18-00496 S of the Czech Science Foundation. V.C. is grateful to the University of Adelaide for its hospitality and support. V.C and T.L. thank the mathematical research institute MATRIX in Australia where the first version of the paper was completed. 
allows to relate and apply several fundamental results in differential geometry: Berger's list of irreducible Riemannian holonomy groups [8] and the classification of those that belong to manifolds with parallel spinors by Wang [30], the understanding of the geometric structures that correspond to these holonomy groups, and finally Gallot's Theorem [17] that the cone $(\widetilde{M}, \breve{g})$ over a complete manifold $(M, g)$ is either flat or irreducible. This result allows to determine the geometry of $(M, g)$ : if the cone $(\widetilde{M}, \breve{g})$ is flat, then $(M, g)$ has constant sectional curvature 1 , and if the cone is irreducible, the geometry of $(M, g)$ is determined by the special holonomy of the cone (Ricci-flat Kähler, hyper-Kähler, or exceptional).

One of the motivations to study semi-Riemannian cones is the Killing spinor equation on semi-Riemannian manifolds, but indefinite cones already become relevant in the Riemannian context. Indeed, imaginary Killing spinors on a Riemannian manifold $(M, g)$ correspond to parallel spinors on the time-like cone

$$
\left(\widehat{M}=\mathbb{R}^{>0} \times M, \widehat{g}=-\mathrm{d} r^{2}+r^{2} g\right) .
$$

Riemannian manifolds with imaginary Killing spinors were classified by Baum in [6, 5] without using the cone construction, but our results about Lorentzian cones in [1] allow to reprove Baum's classification.

Another motivation stems from supergravity (and string theory), where semi-Riemannian cones play a two-fold role. One the one hand, they appear as scalar geometries (of arbitrary dimension) in the superconformal formulation of supergravity theories, on the other hand, they can be used to study space-times which are part of supersymmetric solutions of the equations of motion of theories of (Poincaré) supergravity or of string theories. In the latter case, the supersymmetry equations can be analysed by passing to the time-like cone over the Lorentzian space-time manifold, which is a semi-Riemannian cone of index 2.

A generalisation of Bär's method to indefinite semi-Riemannian manifolds has two aspects: a holonomy classification of indefinite semi-Riemannian cones and the description of the corresponding geometry of the base. Both tasks face several difficulties in the semiRiemannian context. The fundamental difficulty is that for metrics of arbitrary signature the holonomy group may not act completely reducibly: there are semi-Riemannian manifolds whose holonomy group admits an invariant subspace that is degenerate for the metric. As a consequence, those manifolds cannot be decomposed into a product of manifolds with irreducible holonomy, as it is the case for Riemannian manifolds. Hence, in an indefinite semi-Riemannian context, irreducibility has to be replaced by indecomposability. A semiRiemannian manifold is indecomposable if its holonomy representation (i.e., the representation of the holonomy algebra on the tangent space) does not admit an invariant subspace that is non-degenerate for the metric. By the splitting theorems of de Rham [10] and $\mathrm{Wu}$ 31, such metrics do not have a local decomposition into product metrics, hence the term indecomposable. Therefore, a generalisation of Bär's method to semi-Riemannian geometry requires two steps:

(A) Generalise Gallot's Theorem to the case of semi-Riemannian cones.

(B) For indecomposable semi-Riemannian cones, describe the holonomy of the cone and the local geometry of the base.

The problem in (A) was solved in [1], where we studied decomposable indefinite semiRiemannian cones and obtained a generalisation of Gallot's result. In fact we showed that a 
cone over a complete and compact semi-Riemannian manifold is either flat or indecomposable. The results in [1] have been generalised in the compact and in the complete case in [23, 22, 24]. Further results about decomposable cones have been obtained in [12, Theorems 5 and 6]. Cones over Lorentzian Sasaki manifolds and their holonomy were studied in the decomposable and indecomposable case in [15].

1.2. Results. In this article we deal with problem (B), i.e., we study the local geometry of the base and the holonomy of the cone in the case when the cone is indecomposable. This setting naturally splits into two different scenarios: the holonomy of the cone is irreducible, or it admits an invariant subspace that is totally null but no non-degenerate invariant subspace. The irreducible case is well understood as there is Berger's classification of irreducible holonomy groups [8, which we describe in Section 2.2 with the following result:

Theorem 1.1. If $(\widehat{M}, \widehat{g})$ is a time-like cone with irreducible holonomy algebra $\mathfrak{g}$, then $\mathfrak{g}$ is isomorphic to one of the following Lie algebras

$$
\begin{array}{rlrlrl}
\mathfrak{s o}(t, s), & \mathfrak{u}(p, q), \mathfrak{s u}(p, q) & \subset & \mathfrak{s o}(2 p, 2 q), & \mathfrak{s p}(p, q) & \subset \mathfrak{s o}(4 p, 4 q), \\
\mathfrak{s o}(n, \mathbb{C}) \quad \subset \quad \mathfrak{s o}(n, n), & \mathfrak{g}_{2}^{\mathbb{C}} & \subset \mathfrak{s o}(7,7), & \mathfrak{s p i n}(7, \mathbb{C}) & \subset \mathfrak{s o}(8,8), \\
\mathfrak{g}_{2} & \subset \mathfrak{s o}(7), & \mathfrak{s p i n}(7) & \subset \mathfrak{s o}(8), \\
\mathfrak{g}_{2(2)} & \subset \mathfrak{s o}(3,4), & \mathfrak{s p i n}(3,4) & \subset \mathfrak{s o}(4,4) .
\end{array}
$$

More interesting is the non-irreducible indecomposable case. Here the cone admits a totally null vector distribution of rank $k>0$ that is invariant under parallel transport, or equivalently, its space of sections is invariant under differentiation with respect to the Levi-Civita connection. In general, this case is rather difficult and no general holonomy classification is known. However, the parallel vector distribution determines the local structure of the base. This became obvious in [1] where we studied the case of Lorentzian indecomposable cones. As mentioned, some of our motivation comes from the equations of motion of supersymmetric theories of gravity, where the space-time metric is Lorentzian (that is of index 1). Hence we will focus on cones that have index 2 , that is signature $(2, n-2)$. For these the totally null parallel vector distribution is of rank 1, i.e., a null line, or of rank 2 , i.e., a null plane. Many of our results will however hold for cones in arbitrary signature but with an invariant null line or null plane.

In Section 3 we will study the case of a parallel null line, and describe the local structure of the base as well as of the cone:

Theorem 1.2. Let $(\widehat{M}, \widehat{g})$ be the time-like cone over a semi-Riemannian manifold $(M, g)$. If the cone admits a parallel null line field $\mathbf{L}$, then locally there is a parallel trivializing section of $\mathbf{L}$. Moreover, on a dense open subset $\widehat{M}_{\mathrm{reg}} \subset \widehat{M}$, the metric $\widehat{g}$ is locally isometric to a warped product of the form

$$
\widetilde{g}_{0}=2 \mathrm{~d} u \mathrm{~d} v+u^{2} g_{0}
$$

with a semi-Riemannian metric $g_{0}$, and the metric $g$ is locally of the form

$$
g=\mathrm{d} s^{2}+\mathrm{e}^{2 s} g_{0}
$$


In the case when the above decompositions hold globally (see Theorem 3.5), the situation can be summarised in the commutative diagram:

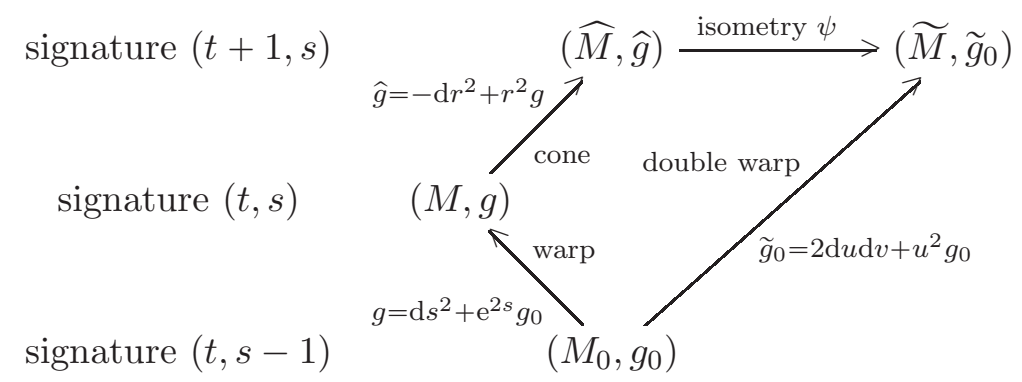

Here $\widetilde{M}=\mathbb{R}^{+} \times \mathbb{R}^{-} \times M_{0}$, see (3.1) for the definition of $\psi$. This result motivates the study of metrics of the form (1.3) in Section 4. Such metrics have a parallel null vector field $\partial_{v}$ and it was shown in [21] that their holonomy algebra $\tilde{\mathfrak{g}}=\mathfrak{h o l}\left(\widetilde{g}_{0}\right)$ is contained in $\mathfrak{h o l}\left(g_{0}\right) \ltimes \mathbb{R}^{t, s}$, where $(t, s)$ is the signature of the metric $g_{0}$, and moreover that $\operatorname{pr}_{\mathfrak{s o}(n)}(\tilde{\mathfrak{g}})=\mathfrak{h o l}\left(g_{0}\right)$. For a Lorentzian metric $\widetilde{g}$, i.e., when $g_{0}$ is Riemannian, it was shown in [21, 1] that we have in fact

$$
\tilde{\mathfrak{g}}=\mathfrak{h o l}\left(g_{0}\right) \ltimes \mathbb{R}^{n},
$$

which means that the holonomy of the cone is determined solely by the holonomy of the metric $g_{0}$. In higher signatures, i.e., when $g_{0}$ is not Riemannian, this is no longer true, as examples will show. Our approach is to consider the ideal of translations in $\mathfrak{h o r}\left(\widetilde{g}_{0}\right)$,

$$
T:=\mathfrak{h o l}\left(\widetilde{g}_{0}\right) \cap \mathbb{R}^{t, s},
$$

and use this for a first, purely algebraic study of indecomposable subalgebras in the stabiliser of a null vector. This will be carried out in Section 5.1, which is the most technical section of the paper. The key observation is that

$$
\tilde{\mathfrak{g}} / T=\left\{(X, \varphi(X)) \mid X \in \mathfrak{h o l}\left(g_{0}\right)\right\}, \quad \text { with } \varphi \in Z^{1}\left(\mathfrak{h o l}\left(g_{0}\right), \mathbb{R}^{t, s} / T\right),
$$

where $Z^{1}\left(\mathfrak{h o l}\left(g_{0}\right), \mathbb{R}^{t, s} / T\right)$ denotes the cocycles of $\mathfrak{h o l}\left(g_{0}\right)$ with values in $\mathbb{R}^{t, s} / T$. For example, in order to obtain results for time-like cones over Lorentzian manifolds, we will compute $Z^{1}\left(\mathfrak{g}, \mathbb{R}^{1, n-1} / T\right)$, for indecomposable subalgebras $\mathfrak{g}$ of $\mathfrak{s o}(1, n-1)$ (these belong to one of four types according to [7]).

In Section 6 we apply these algebraic results to obtain the following result.

Theorem 1.3. Let $g_{0}$ be a Lorentzian metric on an $n$-dimensional simply connected manifold $M$ and $\widetilde{g}_{0}$ the metric of signature $(2, n)$ on $\mathbb{R}^{+} \times \mathbb{R} \times M$ defined in (1.3). If the holonomy of $\widetilde{g}_{0}$ acts indecomposably and with invariant null line, then

$$
\mathfrak{h o r}\left(\widetilde{g}_{0}\right)=\mathfrak{h o l}\left(g_{0}\right) \ltimes \mathbb{R}^{1, n-1},
$$

or $g_{0}$ admits a parallel null vector field and $\widetilde{g}_{0}$ admits two linearly independent parallel null vector fields that are orthogonal to each other.

This theorem shows that if the holonomy of $\widetilde{g}_{0}$ is not equal to the semi-direct product $\mathfrak{h o l}\left(g_{0}\right) \ltimes \mathbb{R}^{1, n-1}$, then $\widetilde{g}_{0}$ and hence the cone admits a parallel null plane (which in addition is spanned by two parallel null vector fields). We study the case of cones admitting a totally null parallel 2-plane in the remainder of the article. In Section 7 we show: 
Theorem 1.4. If the timelike cone $(\widehat{M}, \widehat{g})$ over a semi-Riemannian manifold $(M, g)$ admits a parallel, totally null 2-plane field, then, locally over an open dense subset the base $(M, g)$ admits two vector fields $V$ and $Z$ satisfying

$$
g(V, V)=0, \quad g(Z, Z)=1, \quad g(V, Z)=0,
$$

and such that

$$
\begin{aligned}
& \nabla_{X} V=\alpha(X) V+g(X, V) Z, \\
& \nabla_{X} Z=-X+\beta(X) V+g(X, Z) Z,
\end{aligned}
$$

with 1 -forms $\alpha$ and $\beta$ on $M$. In particular, the base $(M, g)$ admits a geodesic, shearfree null congruence defined by $V$.

Conversely, each pair of vector fields $V$ and $Z$ on $M$ satisfying relations (1.5), (1.6) and (1.7) defines a parallel distribution of totally null 2-planes on the cone.

Note that equation (1.6) implies that $V^{\perp}$ is integrable. This allows us to determine the local form of the metrics with vector field $V$ and $Z$ satisfying equations (1.5 1.7):

Theorem 1.5. A semi-Riemannian metric $(M, g)$ admits vector fields $V$ and $Z$ with (1.5) 1.7) if and only if $(M, g)$ is locally of the form $M=M_{0} \times \mathbb{R}^{3}$ and

$$
g=\mathrm{d} s^{2}+e^{-2 s} g_{0}(u)+2 \mathrm{~d} u \eta,
$$

for a family of metrics $g_{0}(u)$ on $M_{0}$ depending on $u$ and a 1-form $\eta$ on $M$ such that $\eta\left(\partial_{t}\right)$ is nowhere vanishing satisfying the following system of first order PDEs:

$$
\begin{aligned}
\partial_{t} \eta_{t}=\partial_{s} \eta_{t}=X \eta_{t} & =0 \\
\partial_{t} \eta_{s} & =2 \eta_{t}, \\
\partial_{t}(\eta(X)) & =0, \\
\partial_{s} \eta(X)-X \eta_{s} & =-2 \eta(X)
\end{aligned}
$$

for all $X \in \mathfrak{X}\left(M_{0}\right)$ and where $\eta_{t}:=\eta\left(\partial_{t}\right)$ and $\eta_{s}:=\eta\left(\partial_{s}\right)$.

Finally we give explicitly the general solution for the system (1.8), providing us with a construction method of metrics whose cone admits a totally null two plane.

Acknowledgements. We would like to thank the anonymous referee for many valuable comments, in particular about the proof of Theorem 5.7, and for pointing out to us the result of Corollary 7.10 .

\section{Preliminaries}

2.1. Fundamental properties of time-like cones. Let $(M, g)$ be a semi-Riemannnian manifold and $\widehat{M}:=\mathbb{R}^{+} \times M$ with the metric

$$
\widehat{g}:=-\mathrm{d} r^{2}+r^{2} g
$$

be the time-like cone or just the cone over $(M, g)$. We denote by

$$
\xi=r \frac{\partial}{\partial r}
$$


the Euler vector field. The Levi-Civita connection $\hat{\nabla}$ of $\hat{g}$ reduces to the Levi-Civita connection $\nabla$ of $g$ in the following way

$$
\begin{aligned}
\hat{\nabla} \xi & =\mathrm{Id}, \\
\hat{\nabla}_{X} Y & =\nabla_{X} Y+g(X, Y) \xi,
\end{aligned}
$$

where here and in the following formulas $X, Y, Z \in \mathfrak{X}(M)$, and the curvature is given as

$$
\begin{aligned}
\xi\lrcorner \widehat{R} & =0, \\
\widehat{R}(X, Y) Z & =R(X, Y) Z+g(Y, Z) X-g(X, Z) Y .
\end{aligned}
$$

Hence, for the Ricci tensor we obtain that

$$
\begin{aligned}
\xi\lrcorner \widehat{\operatorname{Ric}} & =0, \\
\widehat{\operatorname{Ric}}(X, Y) & =\operatorname{Ric}(X, Y)+(n-1) g(X, Y) .
\end{aligned}
$$

This leads to the following observations:

Proposition 2.1. Let $(\widehat{M}, \widehat{g})$ be the cone over $(M, g)$.

(1) $(M, g)$ has constant curvature -1 if and only if the cone $(\widehat{M}, \widehat{g})$ is flat.

(2) If $(\widehat{M}, \widehat{g})$ is Einstein, then it is Ricci-flat.

(3) If $(M, g)$ is Einstein with Ric $=(1-n) g$, then $(\widehat{M}, \widehat{g})$ is Ricci-flat.

Finally we recall the important known fact that the existence of a time-like vector field $\xi$ with $\nabla \xi=$ Id characterises cones locally, see for example [18] or [12, Lemma 1]. We include the proof here for expository reasons.

Proposition 2.2. Let $(\widehat{M}, \widehat{g})$ be a semi-Riemannian manifold of dimension $n+1$ that admits a time-like vector field $\xi$ such that $\hat{\nabla} \xi=\mathrm{Id}$. Then there are local coordinates $\left(r, x^{1}, \ldots x^{n}\right)$ such that $\hat{g}$ is of the form

$$
\widehat{g}=-\mathrm{d} r^{2}+r^{2} g_{i j}\left(x^{1}, \ldots, x^{n}\right) \mathrm{d} x^{i} \mathrm{~d} x^{j},
$$

where $i, j$ run from 1 to $n$, we use the Einstein summation convention, and $g_{i j}=g_{i j}\left(x^{1}, \ldots, x^{n}\right)$ are functions of the $x^{k}$ coordinates only.

Proof. The vector field $\xi$ defines a positive function $r$ via

$$
\widehat{g}(\xi, \xi)=-r^{2} .
$$

Differentiating this relation gives

$$
2 r \mathrm{~d} r=\mathrm{d}\left(r^{2}\right)=-\mathrm{d}(\widehat{g}(\xi, \xi))=-2 g(\xi, \cdot)=-2 \xi^{b},
$$

where the musical isomorphism $b$ denotes the metric dual with respect to $\widehat{g}$. Hence

$$
\xi^{b}=-\mathrm{d}\left(\frac{r^{2}}{2}\right)
$$

is exact and therefore $\xi=-\widehat{\nabla} \frac{r^{2}}{2}$ is a gradient vector field. The level sets of the function $r$ are orthogonal to $\xi$ and we can fix coordinates $\left(x^{1}, \ldots, x^{n}\right)$ on the level sets such that $\left(r, x^{1}, \ldots, x^{n}\right)$ are local coordinates on $\widehat{M}$. In these coordinates the metric has the form

$$
g=-\mathrm{d} r^{2}+\hat{g}_{i j}\left(r, x^{1}, \ldots, x^{n}\right) \mathrm{d} x^{i} \mathrm{~d} x^{j},
$$


and it holds $\xi=r \partial_{r}$. Since $\hat{\nabla} \xi=\mathrm{Id}$, the vector field $\xi$ is a homothety,

$$
\mathcal{L}_{\xi} \widehat{g}=2 \widehat{g},
$$

which implies that

$$
\widehat{g}_{i j}\left(r, x^{1}, \ldots, x^{n}\right)=r^{2} g_{i j}\left(x^{1}, \ldots, x^{n}\right)
$$

for some functions $g_{i j}\left(x^{1}, \ldots, x^{n}\right)$ of the $x^{i}$ coordinates.

2.2. The holonomy of irreducible cones. For irreducible cones the possible holonomy groups are known from the Berger list [8, which comprises the orthogonal algebra and the three lists (2.5 2.7) below. In the following let $\mathfrak{h} \subset \mathfrak{s o}(t+1, q)$ the irreducible holonomy algebra of a semi-Riemannian manifold $(\widehat{M}, \widehat{g})$, i.e., one of the entries in Berger's list. For each possible $\mathfrak{h}$ we will now determine if it can be the holonomy algebra of a cone.

(1) $\mathfrak{h}=\mathfrak{s o}(t+1, s)$ : This is the holonomy algebra of a generic semi-Riemannian manifold of signature $(t+1, s)$.

Proposition 2.3. Let $(M, g)$ be a semi-Riemannian manifold of signature $(t, s)$ and of constant curvature $\kappa \neq-1$ and let $(\widehat{M}, \widehat{g})$ be the time-like cone over $(M, g)$. Then $\mathfrak{h o r}(\widehat{M}, \widehat{g})=\mathfrak{s o}(t+1, s)$.

Proof. The curvature endomorphisms of $(M, g)$ are of the form

$$
R(X, Y)=\kappa(g(Y, \cdot) X-g(X, \cdot) Y) .
$$

Since the holonomy algebra contains all curvature endomorphisms, equation (2.3) shows that

$$
\mathfrak{s o}(t, s) \subset \mathfrak{h o l}(\widehat{M}, \widehat{g}),
$$

where $\mathfrak{s o}(t, s)$ is embedded as the stabiliser of the vector $\xi$. Moreover, equations (2.2 2.3) show that

$$
\left(\hat{\nabla}_{X} \widehat{R}\right)(Y, Z) \xi=-\widehat{R}(X, Y) Z=-2(g(Y, Z) X-g(X, Z) Y) .
$$

This establishes $\mathfrak{h o r}(\widehat{M}, \widehat{g})=\mathfrak{s o}(t+1, s)$.

$(2) \mathfrak{h}$ is the holonomy of an irreducible symmetric space or one of the following algebras:

$$
\begin{aligned}
& \mathfrak{s p}(1) \oplus \mathfrak{s p}(p, q) \quad \subset \quad \mathfrak{s o}(2 p, 2 q), \\
& \mathfrak{s l}(2, \mathbb{R}) \oplus \mathfrak{s p}(m, \mathbb{R}) \subset \mathfrak{s o}(2 m, 2 m), \\
& \mathfrak{s l}(2, \mathbb{C}) \oplus \mathfrak{s p}(m, \mathbb{C}) \subset \mathfrak{s o}(4 m, 4 m),
\end{aligned}
$$

where $p+q$ and $m$ are $>1$. In the first case the metric is quaternionic Kähler of signature $(4 p, 4 q)$ and in the second it is quaternionic para-Kähler. Examples of the third type are obtained by complexifying manifolds with holonomy of the first two types, as discussed below. In these examples $(\widehat{M}, \widehat{g})$ is Einstein with nonzero Einstein constant, see [2, Theorem 3]. Hence, these cases can be excluded as holonomy of cones by Proposition 2.1.

$(3) \mathfrak{h}$ is one of the following:

$$
\begin{aligned}
\mathfrak{u}(p, q), \mathfrak{s u}(p, q) & \subset \mathfrak{s o}(2 p, 2 q), & \mathfrak{s p}(p, q) & \subset \mathfrak{s o}(4 p, 4 q), \\
\mathfrak{g}_{2} & \subset \mathfrak{s o}(7), & \mathfrak{s p i n}(7) & \subset \mathfrak{s o}(8), \\
\mathfrak{g}_{2(2)} & \subset \mathfrak{s o}(3,4), & \mathfrak{s p i n}(3,4) & \subset \mathfrak{s o}(4,4) .
\end{aligned}
$$


The geometric structures corresponding to these algebras do exist on cones over semi-Riemannian manifolds with certain structures. In fact, the following relations between structure on the base $(M, g)$ and on the cone are well known (see for example 44 for the Riemannian case and [20] for the indefinite cases, and references therein):

(i) The cone over a (semi-Riemannian) Sasaki, Einstein-Sasaki or 3-Sasaki manifold is, respectively, a Kähler, Ricci-flat Kähler or hyper-Kähler manifold and hence has holonomy contained in $\mathfrak{u}(p, q), \mathfrak{s u}(p, q)$ or $\mathfrak{s p}(p, q)$.

(ii) The cone over a strict nearly-Kähler manifold of dimension 6, Riemannian or of signature $(2,4)$, has a parallel $\mathbf{G}_{2^{-}}$or $\mathbf{G}_{2(2)}$-structure and hence has holonomy contained in $\mathfrak{g}_{2}$ or $\mathfrak{g}_{2(2)}$. Similarly, the cone over a nearly para-Kähler manifold with $|\nabla J|^{2} \neq 0$ has holonomy contained in $\mathfrak{g}_{2(2)}$, see [9, Prop. 3.1].

(iii) The cone over a 7 -manifold with a nearly-parallel $\mathbf{G}_{2}$-structure, Riemannian or of signature $(3,4)$, has a parallel $\operatorname{Spin}(7)$ - or $\operatorname{Spin}(3,4)$-structure and hence has holonomy contained in $\mathfrak{s p i n}(7)$ or $\mathfrak{s p i n}(3,4)$.

The question remains, whether the holonomy of the cone is not only contained but actually equal to one of the algebras in the list (2.6). In the Riemannian setting (which corresponds to the case where the base of the time-like cone is negative definite) this can be established by using Gallot's Theorem that the (space-like) cone over a complete Riemannian manifold $(M, g)$ is either flat or irreducible and then by constructing a complete $(M, g)$ with the corresponding structure. For indefinite metrics several gaps open up in this argument: our generalisation of Gallot's Theorem in [1] assumes that $(M, g)$ to be compact and complete and implies that the cones is flat or indecomposable, but not necessarily irreducible. Hence, even if one constructed compact and complete indefinite semi-Riemannian manifolds with the above structures, the cone would not have to be irreducible and hence its holonomy could be an indecomposable, non irreducible subalgebra of the algebras in (2.6). We suspect however, that for a "generic" semi-Riemannian manifold with one of the above structures, the cone has holonomy equal to the algebras in (2.6). An explicit way of constructing examples of cones with special holonomy is given below in Remark 2.7.

$(4) \mathfrak{h}$ is one of the following algebras:

$$
\begin{aligned}
\mathfrak{s o}(n, \mathbb{C}) & \subset \mathfrak{s o}(n, n) & \mathfrak{s l}(2, \mathbb{C}) \oplus \mathfrak{s p}(m, \mathbb{C}) & \subset \mathfrak{s o}(4 m, 4 m) \\
\mathfrak{g}_{2}^{\mathbb{C}} & \subset \mathfrak{s o}(7,7), & \mathfrak{s p i n}(7, \mathbb{C}) & \subset
\end{aligned}
$$

Examples can be obtained by complexification as we will explain now in detail. In the case of $\mathfrak{s l}(2, \mathbb{C}) \oplus \mathfrak{s p}(m, \mathbb{C})$ the metric is then Einstein of nonzero scalar curvature (incompatible with a cone), whereas in the two exceptional cases it is Ricci-flat.

Realisation of complex holonomy algebras. Let $(M, g)$ be a connected real analytic manifold endowed with a real analytic semi-Riemannian metric. Then it is easy to see that $M$ can be embedded into a connected complex manifold $M^{\mathbb{C}}$ with the following properties.

(1) There exists an atlas of $M^{\mathbb{C}}$ such that each of its charts $\varphi: U \rightarrow \mathbb{C}^{n}$ is real-valued on $U \cap M$ and the restrictions $\left.\varphi\right|_{U \cap M}: U \cap M \rightarrow \mathbb{R}^{n}, U \cap M \neq \varnothing$, form an atlas of $M$.

(2) The metric coefficients $g_{i j}(x)$ with respect to the real coordinates $x=\left(x^{1}, \ldots, x^{n}\right)=$ $\left.\varphi\right|_{U \cap M}$ are given by real power series converging in $U \cap M$. 
(3) The power series $g_{i j}(z)$ in the holomorphic coordinates $z=\left(z^{1}, \ldots, z^{n}\right)=\varphi$ converges in $U$ for all $i, j$.

It follows that we can define a holomorphic symmetric tensor field $g^{\mathbb{C}}$ on $M^{\mathbb{C}}$ by

$$
\left.g^{\mathbb{C}}\right|_{U}=\sum g_{i j}(z) \mathrm{d} z^{i} \mathrm{~d} z^{j}
$$

The tensor field is non-degenerate on a neighborhood of $M$ and by restriction we can always assume that it is non-degenerate on $M^{\mathbb{C}}$. Then it defines what is called a holomorphic Riemannian metric on $M^{\mathbb{C}}$. We will call $\left(M^{\mathbb{C}}, g^{\mathbb{C}}\right)$ a complexification of $(M, g)$. Recall that a pair consisting of a complex manifold and a holomorphic Riemannian metric on that manifold is called a holomorphic Riemannian manifold. Note that $\left(M^{\mathbb{C}}, g^{\mathbb{C}}\right)$ is unique as a germ of holomorphic Riemannian manifold along $M$.

We define the holonomy algebra of a holomorphic Riemannian manifold $\left(M^{\mathbb{C}}, g^{\mathbb{C}}\right)$ at $p \in M^{\mathbb{C}}$ as the Lie algebra spanned by all the skew-symmetric endomorphisms

$$
\left(\left(\nabla^{\mathbb{C}}\right)_{v_{1}, \ldots, v_{k}}^{k} R^{\mathbb{C}}\right)\left(v_{k+1}, v_{k+2}\right) \in \mathfrak{s o}\left(T_{p}^{1,0} M^{\mathbb{C}}\right) \cong \mathfrak{s o}\left(T_{p} M\right)^{\mathbb{C}},
$$

where $v_{1}, \ldots, v_{k+2} \in T_{p}^{1,0} M^{\mathbb{C}}$ and $k \geqslant 0$. Here $\nabla^{\mathbb{C}}$ denotes the (holomorphic) Levi-Civita connection of $g^{\mathbb{C}}$ and $R^{\mathbb{C}}$ its curvature tensor.

Proposition 2.4. Let $\left(M^{\mathbb{C}}, g^{\mathbb{C}}\right)$ be a complexification of a connected semi-Riemannian manifold $(M, g)$. Then the holonomy algebra of $\left(M^{\mathbb{C}}, g^{\mathbb{C}}\right)$ is given by the complexification $\mathfrak{h}^{\mathbb{C}}$ of the holonomy algebra $\mathfrak{h}$ of $(M, g)$.

Proof. By the Ambrose-Singer theorem for real analytic semi-Riemannian manifolds we know that $\mathfrak{h}$ is spanned by all the endomorphisms $\left(\nabla_{v_{1}, \ldots, v_{k}}^{k} R\right)\left(v_{k+1}, v_{k+2}\right) \in \mathfrak{s o}\left(T_{p} M\right)$, where $v_{1}, \ldots, v_{k+2} \in T_{p} M$ and $k \geqslant 0$. From the definition of $g^{\mathbb{C}}$ as complex-analytic extension of $g$ it is clear that the Levi-Civita connection $\nabla^{\mathbb{C}}$ of $g^{\mathbb{C}}$ coincides with the complex-analytic extension of the Levi-Civita connection $\nabla$ of $g$. The same relation holds for the curvature tensors and their covariant derivatives. This implies the proposition.

Next we consider the real analytic manifold $N$ of dimension $2 n$ underlying the complex manifold $M^{\mathbb{C}}$. It carries a corresponding integrable complex structure $J$ and we can identify $(N, J)$ with $M^{\mathbb{C}}$. We endow $N$ with the real analytic semi-Riemannian metric

$$
g_{N}:=2 \operatorname{Re} g^{\mathbb{C}} .
$$

Note that $g_{N}$ can be considered as a (fibrewise) real bilinear form on $T N$ by means of the canonical identification

$$
T N \cong T^{1,0} N, \quad X \mapsto X^{1,0}=\frac{1}{2}(X-i J X) .
$$

The factor 2 in (2.8) is chosen such that $g^{\mathbb{C}}$ is obtained by restricting (the complex bilinear extension of) $g_{N}$ to $T^{1,0} N$.

We observe that the metric $g_{N}$ can be defined on the real analytic manifold $N$ underlying any holomorphic Riemannian manifold $\left(M^{\mathbb{C}}, g^{\mathbb{C}}\right)$ irrespective of whether $\left(M^{\mathbb{C}}, g^{\mathbb{C}}\right)$ is a complexification of a semi-Riemannian manifold $(M, g)$.

Theorem 2.5. Let $\left(M^{\mathbb{C}}, g^{\mathbb{C}}\right)$ be a connected holomorphic Riemannian manifold and $\left(N, g_{N}\right)$ the corresponding semi-Riemannian manifold. Then $\left(N, g_{N}\right)$ has neutral signature and its holonomy algebra is isomorphic to the holonomy algebra of $\left(M^{\mathbb{C}}, g^{\mathbb{C}}\right)$. 
Proof. Note first that $g_{N}(J \cdot, J \cdot)=-g_{N}$, since $g_{N}$ is of type $(2,0)+(0,2)$ with respect to $J$. This implies that $g_{N}$ has neutral signature, since $J$ it maps a maximal definite subspace of $T_{p} N$ to a maximal definite subspace of the same dimension and of opposite signature.

We consider first the Lie algebra $\mathfrak{s o}\left(T_{p} N\right), p \in N$, with respect to $g_{N}$ and its subalgebra

$$
\mathfrak{s o}\left(T_{p} N\right)^{J}:=\left\{A \in \mathfrak{s o}\left(T_{p} N\right) \mid[A, J]=0\right\} .
$$

The latter can be considered as a complex Lie algebra with the complex structure $A \mapsto J A$. Indeed, $J A$ is $g_{N}$-skew-symmetric as the product of a symmetric with a commuting skewsymmetric endomorphism. The symmetry of $J$ follows from the fact that $J$ is an antiisometry squaring to minus one.

We claim that $\mathfrak{s o}\left(T_{p} N\right)^{J}$ is canonically isomorphic to the complex Lie algebra $\mathfrak{s o}\left(T_{p}^{1,0} N\right)$ with respect to $g^{\mathbb{C}}$. Using the metric $g_{N}$, we can identify $\mathfrak{s o}\left(T_{p} N\right)^{J}$ with the set of real points in $\bigwedge^{2,0} T_{p} N \oplus \bigwedge^{0,2} T_{p} N$ and the latter can be identified with $\bigwedge^{2,0} T_{p} N \cong \bigwedge^{2} T^{1,0} M$ by projecting to the $(2,0)$-component. Finally, using the metric $g^{\mathbb{C}}$, we can identify $\wedge^{2} T^{1,0} M$ with $\mathfrak{s o}\left(T_{p}^{1,0} N\right)$. This yields a canonical isomorphism

$$
\Phi: \mathfrak{s o}\left(T_{p} N\right)^{J} \rightarrow \mathfrak{s o}\left(T_{p}^{1,0} N\right)
$$

of complex vector spaces. It simply maps $A \in \mathfrak{s o}\left(T_{p} N\right)^{J}$ to its restriction to $T^{1,0} N$. Therefore it is even an isomorphism of Lie algebras.

Next we show, for all $v_{1}, \ldots v_{k+2} \in T_{p} N$, that under the canonical isomorphism (2.9) the tensor $\left(\nabla^{N}\right)_{v_{1}, \ldots, v_{k}}^{k} R^{N}\left(v_{k+1}, v_{k+2}\right)$ is mapped to $\left(\nabla^{\mathbb{C}}\right)_{w_{1}, \ldots, w_{k}}^{k} R^{\mathbb{C}}\left(w_{k+1}, w_{k+2}\right)$, where $w_{j}=$ $v_{j}^{1,0}, \nabla^{N}$ denotes the Levi-Civita connection of $g_{N}$ and $R^{N}$ its curvature. This implies the theorem, in virtue of the Ambrose-Singer theorem. First we show that $\nabla^{N}$ can be constructed from the holomorphic connection $\nabla^{\mathbb{C}}$. Let $\nabla^{\prime}$ be the unique connection in $(T N)^{\mathbb{C}}$ with the following properties:

(1) $\nabla_{Z}^{\prime} W=\nabla_{Z}^{\mathbb{C}} W$ for all holomorphic vector fields $Z, W$ on $M^{\mathbb{C}}$.

(2) $\nabla_{\bar{Z}}^{\prime} W=0$ for all holomorphic vector fields $Z, W$ on $M^{\mathbb{C}}$.

(3) $\nabla^{\prime}$ is real, that is restricts to a connection in $T N$.

Notice that the above properties imply that the subbundles $T^{1,0} N$ and $T^{0,1} N$ are $\nabla^{\prime}$-parallel and, hence, that $\nabla^{\prime} J=0$. Moreover, using these properties, it is straightforward to check that $\nabla^{\prime}$ is metric torsion-free, since $\nabla^{\mathbb{C}}$ is. This implies that $\nabla^{\prime}$ (when considered as a connection in $T N$ ) coincides with the Levi-Civita connection $\nabla^{N}$. As a consequence, we see that $\nabla^{N} J=0$ and thus $\left(\nabla^{N}\right)_{v_{1}, \ldots, v_{k}}^{k} R^{N}\left(v_{k+1}, v_{k+2}\right) \in \mathfrak{s o}\left(T_{p} N\right)^{J}$. Now let $X, Y$ be real vector fields on an open set $U \subset N$ which are infinitesimal automorphisms of $J$. Then we have the formula

$$
\left(\nabla_{X}^{N} Y\right)^{1,0}=\nabla_{X^{1,0}}^{\mathbb{C}} Y^{1,0}
$$

This follows immediately from the defining properties of $\nabla^{\prime}=\nabla^{N}$ by decomposing $X=$ $Z+\bar{Z}$ and $Y=W+\bar{W}$, where $Z=X^{1,0}, W=Y^{1,0}$ are holomorphic. From (2.10) we deduce that

$$
\left(\left(\left(\nabla^{N}\right)_{v_{1}, \ldots, v_{k}}^{k} R^{N}\left(v_{k+1}, v_{k+2}\right)\right) v_{k+3}\right)^{1,0}=\left(\left(\nabla^{\mathbb{C}}\right)_{w_{1}, \ldots, w_{k}}^{k} R^{\mathbb{C}}\left(w_{k+1}, w_{k+2}\right)\right) w_{k+3},
$$


for all $v_{1}, \ldots, v_{k+3} \in T_{p} N$, where we recall that $w_{j}=v_{j}^{1,0}$. Since the left-hand side is precisely

$$
\Phi\left(\left(\nabla^{N}\right)_{v_{1}, \ldots, v_{k}}^{k} R^{N}\left(v_{k+1}, v_{k+2}\right)\right) w_{k+3}
$$

we can conclude that

$$
\Phi\left(\left(\nabla^{N}\right)_{v_{1}, \ldots, v_{k}}^{k} R^{N}\left(v_{k+1}, v_{k+2}\right)\right)=\left(\nabla^{\mathbb{C}}\right)_{w_{1}, \ldots, w_{k}}^{k} R^{\mathbb{C}}\left(w_{k+1}, w_{k+2}\right),
$$

finishing the proof.

This leads to the following consequence:

Corollary 2.6. The complex holonomies

$$
\mathfrak{s o}(n, \mathbb{C}) \subset \mathfrak{s o}(n, n), \quad \mathfrak{g}_{2}^{\mathbb{C}} \subset \mathfrak{s o}(7,7), \quad \mathfrak{s p i n}(7, \mathbb{C}) \subset \mathfrak{s o}(8,8),
$$

are holonomy algebras of time-like cones.

Proof. This follows from the above considerations and from the fact that the compact real forms of the complex holonomy algebras in (2.11) can be realised by timelike cones over negative definite manifolds. Indeed, if $(\widehat{M}, \widehat{g})$ is a time-like cone with holonomy $\mathfrak{s o}(n)$, $\mathfrak{g}_{2} \subset \mathfrak{s o}(7)$, or $\mathfrak{s p i n}(7) \subset \mathfrak{s o}(8)$, then there is the Euler vector field $\xi \in \Gamma(T \widehat{M})$. Hence the real analytic metric $\widehat{g}^{\mathbb{C}}$ on $\widehat{M}^{\mathbb{C}}$ has the holomorphic Euler vector field $\xi^{\mathbb{C}}$ with $\hat{\nabla}^{\mathbb{C}} \xi^{\mathbb{C}}=$ Id. On the real manifold $N=\widehat{M}^{\mathbb{C}}$ we then have that $\eta=2 \operatorname{Re} \xi^{\mathbb{C}}$ satisfies $\nabla^{N} \eta=$ Id, as a consequence of equation (2.10) applied here to $N=\widehat{M}^{\mathbb{C}}$ instead of $M^{\mathbb{C}}$. By Proposition 2.2 we then get that $N$ is locally a cone, which by Theorem 2.5 has one of the complex holonomies in (2.11) as holonomy algebra.

This proof can be made more explicit in local coordinates. Locally the metric $\widehat{g}$ is of the form

$$
\widehat{g}=-\mathrm{d} r^{2}+r^{2} g_{i j}\left(x^{k}\right) \mathrm{d} x^{i} \mathrm{~d} x^{j}
$$

with Euler vector field $\xi=r \partial_{r} \in \Gamma(T \widehat{M})$. The analytic metric $\widehat{g}^{\mathbb{C}}$ on $\widehat{M}^{\mathbb{C}}$ then is of the form

$$
\widehat{g}^{\mathbb{C}}=-\mathrm{d} u^{2}+u^{2} g_{i j}\left(z^{k}\right) \mathrm{d} z^{i} \mathrm{~d} z^{j}
$$

with coordinates $\left(u=r+i s, z^{1}, \ldots, z^{n}\right)$ with $z^{k}=x^{k}+\mathrm{i} y^{k}$ and holomorphic Euler vector field $\xi^{\mathbb{C}}=u \partial_{u}$ with $\hat{\nabla}^{\mathbb{C}} \xi^{\mathbb{C}}=$ Id. Then the metric $\widehat{h}=\frac{1}{2} g_{N}$ on $N=\widehat{M}^{\mathbb{C}}$ is given by

$$
\widehat{h}=-\mathrm{d} r^{2}+\mathrm{d} s^{2}+\left(r^{2}-s^{2}\right) \operatorname{Re}\left(g_{i j}\left(z^{k}\right) \mathrm{d} z^{i} \mathrm{~d} z^{j}\right)-2 r s \operatorname{Im}\left(g_{i j}\left(z^{k}\right) \mathrm{d} z^{i} \mathrm{~d} z^{j}\right) .
$$

One can directly check that $\eta=r \partial_{r}+s \partial_{s}$ satisfies $\nabla^{N} \eta=\mathrm{Id}$. Moreover, the cone coordinate with respect to $\widehat{h}$ is given by $\rho=\sqrt{r^{2}-s^{2}}$, which satisfies $\widehat{h}(\eta, \cdot)=-\rho \mathrm{d} \rho$.

Remark 2.7. Finally, we note that it is possible to construct examples of pseudo-Riemannian cones with these holonomies using different real forms of the complexified metrics and Proposition 2.4 and Corollary 2.6. For example $\mathbf{S L}(2, \mathbb{R}) \times \mathbf{S L}(2, \mathbb{R})$ admits a unique left-invariant nearly pseudo-Kähler structure, which is a different real form of the complexification of the Riemannian nearly Kähler structure on $\mathbf{S U}(2) \times \mathbf{S U}(2)$, 26]. Hence the cone metrics are different real forms of the holomorphic cone metric. Since the cone over $\mathbf{S U}(2) \times \mathbf{S U}(2)$ has holonomy $\mathfrak{g}_{2}$, the cone over $\mathbf{S L}(2, \mathbb{R}) \times \mathbf{S L}(2, \mathbb{R})$ must have holonomy equal to $\mathfrak{g}_{2(2)}$. 
2.3. Manifolds with parallel null line bundle. In the following manifolds with a parallel null line bundle will be crucial. In this section we will collect some facts about them.

Let $(M, g)$ be a semi-Riemannian manifold with a parallel null line bundle $\mathbf{L}$, i.e., $\mathbf{L}$ is a rank 1 subbundle of $T M$ the fibres of which are null with respect to the metric $g$ and invariant under parallel transport with respect to the Levi-Civita connection $\nabla$ of $g$. This implies that every non-vanishing section $\chi \in \Gamma(\mathbf{L})$ satisfies

$$
\nabla \chi=\alpha \otimes \chi
$$

for a uniquely determined 1-form $\alpha$. Any vector field that satisfies equation (2.12) for some 1 -form $\alpha$ is called a recurrent vector field.

Proposition 2.8. Let $\chi$ be a recurrent vector field on a connected semi-Riemannian manifold $(M, g)$. Then the function $f=g(\chi, \chi)$ is either everywhere positive, negative or zero. In particular, $\chi$ can only have zeros if $f \equiv 0$.

Proof. The equation (2.12) yields the ODE $X(f)=2 \alpha(X) f$ for every vector field $X$. These ODEs imply that if $f$ vanishes at a point, then $f$ vanishes in a neighbourhood of this point. Due to the continuity of $f$ this shows that $M$ is a disjoint union of the three open sets $\{f>0\},\{f<0\}$ and $\{f=0\}$. Now, since $M$ is connected, the proposition follows.

Hence, locally the existence of a parallel null line bundle is equivalent to the existence of a recurrent null vector field, where we recall that a vector field $\chi$ is null if $g(\chi, \chi)=0$ and $\chi$ does not vanish [25, Definition 3 in Chapter 3]. Moreover, a nowhere vanishing recurrent vector field $\chi$ can be rescaled to parallel vector field $\lambda \cdot \chi$, for a non-vanishing function $\lambda$, if and only if the 1 -form $\alpha$ is exact. Indeed, if $\alpha=d h$, then

$$
\nabla\left(\mathrm{e}^{-h} \chi\right)=0
$$

then $\lambda=\mathrm{e}^{-h}$ so that $\lambda \cdot \chi$ is paralle 1 . Conversely, if $\lambda \cdot \chi$ is parallel, then

$$
0=R(X, Y) \chi=\mathrm{d} \alpha(X, Y) \chi
$$

for all $X, Y \in T M$.

Hence, on simply connected manifolds $(M, g)$, nowhere vanishing recurrent vector fields can be rescaled to parallel ones if and only if $\alpha$ is closed. The choice we have when locally choosing a recurrent vector field that spans a null line bundle $\mathbf{L}$ can be used to find special recurrent sections of $\mathbf{L}$.

Lemma 2.9. Let $\mathbf{L}$ be a parallel null line bundle. Then locally there is a recurrent gradient vector field $\chi$ which spans $\mathbf{L}$. This vector field satisfies that $\nabla \chi=h \chi^{b} \otimes \chi$ for a function $h$.

Proof. Since $\mathbf{L}$ is parallel, the hyperplane distribution $\mathbf{L}^{\perp}=\{X \in T M \mid g(X, \mathbf{L})=0$ is parallel and hence involutive. Hence by Frobenius' Theorem $\mathbf{L}^{\perp}$ is integrable and the integral manifolds are given as $f \equiv$ constant for some local function $f$. Hence $\mathbf{L}^{\perp}=\operatorname{ker}(d f)$ and the gradient $\chi:=\operatorname{grad}(f)$ of $f$ spans $\mathbf{L}$. Then $\chi$ is recurrent, i.e., $\nabla \chi=\alpha \otimes \chi$. But then $\chi=\operatorname{grad}(f)$ implies that

$$
0=\mathrm{d} \chi^{b}=\alpha \wedge \chi^{b}
$$

which shows that $\alpha=h \chi^{b}$ for a local function $h$.

\footnotetext{
${ }^{1}$ For non-null recurrent vector fields this shows that they can always be rescaled locally to a parallel vector field, as $0=R(X, Y, \chi, \chi)=\mathrm{d} \alpha(X, Y) g(\chi, \chi)$ yields that $\alpha$ is closed, or more explicitly, $\alpha=\frac{1}{2} \mathrm{~d} \ln f$ with $f=g(\chi, \chi)$ and hence $\frac{1}{\sqrt{f}} \chi$ is parallel.
} 


\section{Cones with parallel null Lines}

In this section we assume that the cone (2.1) over a semi-Riemannian manifold $(M, g)$ admits a null line that is invariant under parallel transport. We will show that locally this implies that the cone admits a parallel null vector field and that the base $(M, g)$ is locally an exponential extension of a semi-Riemannian manifold $\left(M_{0}, g_{0}\right)$, see Definition 3.2 . The total space of the cone will then be shown to be locally isometric to a double warped extension $(\widetilde{M}, \widetilde{g})$ of $\left(M_{0}, g_{0}\right)$, see Definition 3.2 . This will generalise our results for Lorentzian cones in [1, Section 9].

Proposition 3.1. Let $(\widehat{M}, \widehat{g})$ be a timelike cone and assume that $(\widehat{M}, \widehat{g})$ admits a parallel null line $\mathbf{L}$. Then the following holds:

(i) The set $\widehat{M}_{\text {reg }}$ where $\mathbf{L}$ is not perpendicular to the Euler vector field $\xi$ is open and dense and invariant under the flow of $\xi$. So, in particular, $\widehat{M}_{\mathrm{reg}}=\widehat{M_{\mathrm{reg}}}$, where $M_{\text {reg }}:=\widehat{M}_{\text {reg }} \cap M$.

(ii) $\mathbf{L}$ is flat and, hence, locally (and globally if $M$ is simply connected) there is a parallel null vector field that spans $\mathbf{L}$.

Proof. By passing to the universal cover of $(\widehat{M}, \widehat{g})$, that is to the cone over the universal cover of $M$, we can assume that $M$ and $\widehat{M}$ are simply connected. Hence, we can assume that the parallel null line $\mathbf{L}$ is spanned by a nowhere vanishing recurrent vector field $\chi$ on $(\widehat{M}, \widehat{g})$. Then we decompose

$$
\chi=f \xi+Z,
$$

where $Z$ is tangent to $M$ and nowhere vanishing. We claim that the function $f$ cannot vanish on a nonempty open set. If it did, formulae (2.2) would give

$$
\alpha(X) Z=\hat{\nabla}_{X} \chi=\nabla_{X} Z+g(X, Z) \xi
$$

on the open set, and hence $g(X, Z)=0$ for all $X \in T M$, which is a contradiction. This proves that the open set $\widehat{M}_{\text {reg }}=\{p \in \widehat{M} \mid f(p) \neq 0\}$ is dense. The invariance of $\widehat{M}_{\text {reg }}$ under the homothetic flow of $\xi$ follows from the invariance of $\mathbf{L}$ under the flow. The latter is obtained by writing the Lie derivative as $\mathcal{L}_{\xi}=\hat{\nabla}_{\xi}$ - Id and using that $\mathbf{L}$ is parallel.

On $\widehat{M}_{\text {reg }}$ we have

and

$$
\mathrm{d} \alpha(X, \xi) \chi=\widehat{R}(X, \xi) \chi=0
$$

$$
\mathrm{d} \alpha(X, Y) \chi=\widehat{R}(X, Y) \chi=\widehat{R}(X, Y) Z \in T M,
$$

for $X, Y \in T M$. This implies $\mathrm{d} \alpha=0$, since $\widehat{M}_{\text {reg }}$ is dense, proving that $\mathbf{L}$ is flat.

In the next proposition we describe an example of a cone with a parallel null line before showing that every example is locally of this form.

Definition 3.2. Let $\left(M_{0}, g_{0}\right)$ be a semi-Riemannian manifold. Then the warped products $\left(M=\mathbb{R} \times M_{0}, g=d s^{2}+\mathrm{e}^{2 s} g_{0}\right)$ and $\left(\widetilde{M}=\mathbb{R}^{+} \times \mathbb{R}^{-} \times M_{0}, \widetilde{g}=2 \mathrm{~d} u \mathrm{~d} v+u^{2} g_{0}\right)$ will be called the exponential extension and the double warped extension of $\left(M_{0}, g_{0}\right)$, respectively.

Proposition 3.3. Let $\left(M_{0}, g_{0}\right)$ be a semi-Riemannian manifold. The time-like cone $(\widehat{M}, \widehat{g})$ over the exponential extension $(M, g)$ of $\left(M_{0}, g_{0}\right)$ is globally isometric to the double warped extension $(\widetilde{M}, \widetilde{g})$ of $\left(M_{0}, g_{0}\right)$. In particular, the cone admits the parallel null vector field $\partial_{v}$. 
Proof. The cone metric over $(M, g)$ is given by

$$
\widehat{g}=-\mathrm{d} r^{2}+r^{2} \mathrm{~d} s^{2}+r^{2} \mathrm{e}^{2 s} g_{0},
$$

with $r \in \mathbb{R}^{+}$and $s \in \mathbb{R}$. For the diffeomorphism

(3.1) $\psi: \widehat{M}=\mathbb{R}^{+} \times \mathbb{R} \times M_{0} \ni(r, s, p) \longmapsto\left(u=r \mathrm{e}^{s}, v=-\frac{1}{2} r \mathrm{e}^{-s}, p\right) \in \widetilde{M}=\mathbb{R}^{+} \times \mathbb{R}^{-} \times M_{0}$ one checks that

$$
\left(\psi^{-1}\right)^{*} \widehat{g}=2 \mathrm{~d} u \mathrm{~d} v+u^{2} g_{0}
$$

This proves the statement.

Theorem 3.4. Let $(\widehat{M}, \widehat{g})$ be a time-like cone over a semi-Riemannian manifold $(M, g)$. Assume that $(\widehat{M}, \widehat{g})$ admits a parallel null line $\mathbf{L}$. Then the open dense subset $\widehat{M}_{\text {reg }} \subset(\widehat{M}, \widehat{g})$, cf. Proposition 3.1, is locally isometric to the double warped extension $(\widetilde{M}, \widetilde{g})$ of a semiRiemannian manifold $\left(M_{0}, g_{0}\right)$ and the open dense subset $M_{\mathrm{reg}} \subset(M, g)$ is locally isometric to the exponential extension of $\left(M_{0}, g_{0}\right)$.

Proof. Since we have to show the existence of a local isometry, by Proposition 3.1, we can assume that $\mathbf{L}$ admits a parallel trivializing section $\chi$. We write the parallel null vector field $\chi$ on $\widehat{M}$ as

$$
\chi=\hat{f} \xi+\hat{Z}
$$

with $\hat{Z}$ a nowhere vanishing vector field tangent to $M$ and $\hat{f}$ a smooth function on $\widehat{M}$. We will show that $\hat{Z}$ defines vector field $Z$ on $M$. From

$$
[\xi, \hat{Z}]=-d \hat{f}(\xi) \xi+[\xi, \chi]=-d \hat{f}(\xi) \xi-\hat{\nabla}_{\chi} \xi=-d \hat{f}(\xi) \xi-\chi=-(d \hat{f}(\xi)+\hat{f}) \xi-\hat{Z},
$$

with $[\xi, \hat{Z}]$ being tangent to $M$, we get on the one hand that

$$
\mathrm{d} \hat{f}(\xi)+\hat{f}=r \partial_{r}(\hat{f})+\hat{f}=0,
$$

and on the other that

The first equation shows that

$$
[\xi, \hat{Z}]+\hat{Z}=0
$$

$$
\hat{f}=\frac{1}{r} f
$$

with $f$ a function on $M$ and the second that

$$
\hat{Z}=\frac{1}{r} Z
$$

with $Z=r \hat{Z}$ a vector field on $M$, i.e., $[\xi, Z]=0$. Hence we have

$$
\chi=\frac{1}{r}(f \xi+Z) \text {. }
$$

Differentiating in direction of $X \in T M$, by (2.2) we get

$$
0=r \hat{\nabla}_{X} \chi=(d f(X)+g(X, Z)) \xi+f X+\nabla_{X} Z .
$$

which shows that

$$
Z=-\operatorname{grad}(f)
$$

where grad denotes the gradient with respect to $g$, and

$$
\nabla Z=-f \operatorname{Id} .
$$


Hence, the distribution $Z^{\perp}$ on $M$ is integrable and its leafs are given by the level sets of $f$. The vector field $Z$ is not only a gradient but also a conformal vector field, since from (3.2) we compute

$$
\mathcal{L}_{Z} g=-2 f g .
$$

Note also that on $M_{\text {reg }}=\widehat{M}_{\text {reg }} \cap M$, the vector field $Z$ is transversal to the level sets of $f$. This follows from $d f(Z)=g(\operatorname{grad}(f), Z)=-g(Z, Z)=-f^{2}$. Hence, locally on $M_{\text {reg }}$ the metric $g$ is given as

$$
g=\frac{(\mathrm{d} f)^{2}}{f^{2}}+f^{2} g_{0}
$$

where $c^{2} g_{0}$ is the metric $g$ restricted to a level set $\{f=c\}$. Setting $s=\log |f|$ and using Proposition 3.3 this proves the statement in the Theorem.

The local geometry described in this theorem is summarised in diagram (1.4) in the introduction. We also have the following global result.

Theorem 3.5. Let $(\widehat{M}, \widehat{g})$ be a time-like cone over a simply connected and space-like complete semi-Riemannian manifold $(M, g)$. Assume that $(\widehat{M}, \widehat{g})$ admits a parallel null line $\mathbf{L}$ which is nowhere perpendicular to $\xi$. Then $(\widehat{M}, \widehat{g})$ is isometric to the double warped extension $(\widetilde{M}, \widetilde{g})$ of a semi-Riemannian manifold $\left(M_{0}, g_{0}\right)$ and $(M, g)$ is isometric to the exponential extension of $\left(M_{0}, g_{0}\right)$, cf. Definition 3.2.

Proof. Since $M$ (and thus $\widehat{M}$ ) is simply connected, the flat line bundle $\mathbf{L}$ (see Proposition 3.1) admits a parallel section $\chi \neq 0$. By assumption, the function $\widehat{g}(\chi, \xi)$ has no zeroes. As in the proof of Theorem 3.4, we can thus write

$$
\chi=\frac{1}{r}(f \xi+Z)
$$

for a nowhere vanishing function $f$ and $Z=-\operatorname{grad}(f)$ on $M=M_{\text {reg. }}$. Then $Z^{\prime}=\frac{1}{f} Z$ is a space-like geodesic unit vector field, as follows from $g(Z, Z)=f^{2}$ and the equation (3.2):

$$
\nabla_{Z} Z^{\prime}=-\frac{d f(Z)}{f^{2}} Z-Z=0
$$

From the space-like completeness assumption we conclude that $Z^{\prime}$ is complete, giving rise to a global diffeomorphism $M \simeq \mathbb{R} \times M_{0}$ under which the metric takes the form $g=$ $d s^{2}+e^{2 s} g_{0}$.

Remark 3.6. The assumption that $\mathbf{L}$ is nowhere perpendicular to $\xi$ in Theorem 3.5 cannot be dropped. In fact, the universal covering of anti-de Sitter space is simply connected and complete but any parallel line distribution over the time-like cone is somewhere perpendicular to $\xi$. In fact, a complete Lorentzian metric of constant negative curvature cannot be globally written in the form $d s^{2}+e^{2 s} g_{0}$, since the latter metric is incomplete, see [1, Proposition 2.5]. Locally it admits such description, where the Lorentzian metric $g_{0}$ is moreover flat.

In the following we will study metrics of the form $\widetilde{g}=2 \mathrm{~d} u \mathrm{~d} v+u^{2} g_{0}$. For brevity we will drop the index 0 at $g_{0}$. 


\section{Metrics of The FORM $\tilde{g}=2 \mathrm{~d} u \mathrm{~d} v+u^{2} g$}

4.1. Levi-Civita connection, curvature and holonomy. Let $g$ be a semi-Riemannian metric (of signature $(t, s)$ ) on a manifold $M$ of dimension $n$. We want to study the geometry and the holonomy of metrics of signature $(t+1, s+1)$ of the form

$$
\widetilde{g}=2 \mathrm{~d} u \mathrm{~d} v+u^{2} g
$$

from now on to be considered on the maximal domain $\widetilde{M}:=\mathbb{R}^{+} \times \mathbb{R} \times M \supset \mathbb{R}^{+} \times \mathbb{R}^{-} \times M$. Such metrics admit a 2-dimensional solvable group of homotheties given by $(u, v, p) \mapsto$ $\left(\mathrm{e}^{r} u, \mathrm{e}^{r} v+s, p\right)$. Its infinitesimal generators are the parallel null vector field $\partial_{v}$ and the homothetic vector field $U=u \partial_{u}+v \partial_{v}$.

There are obvious inclusions of $M=\{1\} \times\{0\} \times M \subset \widetilde{M}, T M \subset T \widetilde{M}$ and $\Gamma(T M) \subset$ $\Gamma(T \widetilde{M})$. Using these identifications, the Levi-Civita connection of $\widetilde{g}$ can be expressed by

$$
\widetilde{\nabla}_{X} Y=\nabla_{X} Y-u g(X, Y) \partial_{v} \quad \text { and } \quad \tilde{\nabla}_{X} \partial_{u}=\frac{1}{u} X
$$

with $X \in T M, Y \in \Gamma(T M), \nabla$ the Levi-Civita connection of $g$, and all other derivatives either vanish or are determined by the vanishing of the torsion of $\nabla$. Note that the homothetic vector field $U=u \partial_{u}+v \partial_{v}$ satisfies $\tilde{\nabla} U=$ Id. Moreover, for the curvature of $\widetilde{g}$ one computes that

$$
\begin{aligned}
\left.\partial_{v}\right\lrcorner \widetilde{R} & \left.=\partial_{u}\right\lrcorner \widetilde{R}=0, \\
\widetilde{R}(X, Y) Z & =R(X, Y) Z, \quad \text { for all } X, Y, Z \in T M,
\end{aligned}
$$

where $R$ is the curvature tensor of $(M, g)$. Note that this implies for an arbitrary tensor field $Q$ that

$$
\widetilde{\nabla}_{\partial_{u}} \tilde{\nabla}_{X} Q=\tilde{\nabla}_{X} \tilde{\nabla}_{\partial_{u}} Q
$$

for all $X \in \Gamma(T M)$.

For the derivatives of $\widetilde{R}$ we get the following formulae, which determine all possible derivatives. First we observe that

$$
\left(\widetilde{\nabla}_{\partial_{u}} \widetilde{R}\right)\left(\partial_{u}, X\right)=0
$$

for all $X \in T M$. For the $q$-th derivative in $\partial_{u}$-direction we compute

$$
\left(\widetilde{\nabla}_{\partial_{u}} \cdots \widetilde{\nabla}_{\partial_{u}} \widetilde{R}\right)(X, Y) Z=\frac{(-1)^{q}(q+1) !}{u^{q}} R(X, Y) Z
$$

Moreover, a simple induction shows

$$
\begin{aligned}
\left(\widetilde{\nabla}_{X_{1}} \cdots \widetilde{\nabla}_{X_{p}} \widetilde{R}\right)(X, Y) Z= & \left(\nabla_{X_{1}} \cdots \nabla_{X_{p}} R\right)(X, Y) Z \\
& -u \sum_{i=1}^{p}\left(\nabla_{X_{1}} \uparrow \nabla_{X_{p}} R\right)\left(X, Y, Z, X_{i}\right) \partial_{v}
\end{aligned}
$$

for all $X_{i}, X, Y, Z, W \in T M$ and where the symbol.$\uparrow^{i}$. indicates the omission of the $i$ th term. In general, a straightforward computations shows

Proposition 4.1. The $(p+q)$ th derivative of $\widetilde{R}$ is determined by the relations

$$
\begin{aligned}
\left.\partial_{v}\right\lrcorner \widetilde{\nabla}^{k} \widetilde{R} & =0, \\
\left(\widetilde{\nabla}_{\partial_{u}} \widetilde{\nabla}_{X_{1}} \cdots \widetilde{\nabla}_{X_{p}} \widetilde{R}\right)(Y, Z)(W) & =\left(\widetilde{\nabla}_{X_{1}} \widetilde{\nabla}_{\partial_{u}} \widetilde{\nabla}_{X_{2}} \ldots \widetilde{\nabla}_{X_{p}} \widetilde{R}\right)(Y, Z)(W),
\end{aligned}
$$


and the formula

$$
\begin{aligned}
& \left(\widetilde{\nabla}_{\partial_{u}} \cdots \tilde{\nabla}_{\partial_{u}} \tilde{\nabla}_{X_{1}} \cdots \tilde{\nabla}_{X_{p}} \widetilde{R}\right)(X, Y) Z= \\
& \quad=\frac{c(p, q)}{u^{q}}\left(\left(\nabla_{X_{1}} \cdots \nabla_{X_{p}} R\right)(X, Y) Z-u \sum_{i=1}^{p}\left(\nabla_{X_{1}} \stackrel{{ }^{i}}{\uparrow} \nabla_{X_{p}} R\right)\left(X, Y, Z, X_{i}\right) \partial_{v}\right),
\end{aligned}
$$

where $c(p, 0)=1$ and $c(p, q)=(-1)^{q}(p+2) \cdot \ldots \cdot(p+q+1)$ when $q \geqslant 1$.

Our aim is to study the holonomy of metrics $\widetilde{g}=2 \mathrm{~d} u \mathrm{~d} v+u^{2} g$. Since $\partial_{v}$ is a parallel vector field on $(\widetilde{M}, \widetilde{g})$, the holonomy of $(\widetilde{M}, \widetilde{g})$ is contained in the stabiliser of the vector $\partial_{v}$ at a point. By splitting $T \widetilde{M}=\mathbb{R} \partial_{v} \oplus T M \oplus \mathbb{R} \partial_{u}$, where $\operatorname{span}\left\{\partial_{v}, \partial_{u}\right\}=T M^{\perp}$, and fixing an orthonormal basis in $T_{p} M$ we can identify $\mathfrak{s o}\left(T_{p} M, g\right) \simeq \mathfrak{s o}(t, s)$ and have $\mathfrak{h o r}(M, g) \subset$ $\mathfrak{s o}(t, s)$. Hence, we can identify the stabiliser of $\partial_{v}$ in $\mathfrak{s o}(t+1, s+1)$ with $\mathfrak{s o}(t+1, s+1)_{\partial_{v}}=$ $\mathfrak{s o}(t, s) \ltimes \mathbb{R}^{t, s}$ and we get that

$$
\mathfrak{h o l}(\widetilde{M}, \widetilde{g}) \subset \mathfrak{s o}(t, s) \ltimes \mathbb{R}^{t, s}=\left\{\left(\begin{array}{ccc}
0 & g(w, .) & 0 \\
0 & A & -w \\
0 & 0 & 0
\end{array}\right) \mid A \in \mathfrak{s o}(t, s), w \in \mathbb{R}^{t, s}\right\},
$$

where the matrices are with respect to the splitting $T \widetilde{M}=\mathbb{R} \partial_{v} \oplus T M \oplus \mathbb{R} \partial_{u}$ and the identification $T_{p} M=\mathbb{R}^{t, s}$. With these identifications, there are two projections

$$
\operatorname{pr}_{\mathfrak{s o}(t, s)}: \mathfrak{h o l}(\widetilde{M}, \widetilde{g}) \rightarrow \mathfrak{s o}(t, s), \quad \operatorname{pr}_{\mathbb{R}^{t, s}}: \mathfrak{h o r}(\widetilde{M}, \widetilde{g}) \rightarrow \mathbb{R}^{t, s},
$$

to the linear part $A$ and the translational part $w$ in (4.8) of $\mathfrak{s o}(t+1, s+1)_{\partial_{v}}=\mathfrak{s o}(t, s) \ltimes \mathbb{R}^{t, s}$. Since derivatives of the curvature are contained in the holonomy algebra, Proposition 4.1 implies that

$$
\begin{aligned}
\left.\operatorname{pr}_{\mathfrak{s o}(t, s)}\left(\widetilde{\nabla}_{\partial_{u}}^{q} \widetilde{\nabla}_{X_{1}} \cdots \widetilde{\nabla}_{X_{p}} \widetilde{R}\right)(Y, Z)\right) & =\frac{c}{u^{q}}\left(\nabla_{X_{1}} \cdots \nabla_{X_{p}} R\right)(Y, Z) \\
\left.\operatorname{pr}_{\mathbb{R}^{t, s}}\left(\widetilde{\nabla}_{\partial_{u}}^{q} \widetilde{\nabla}_{X_{1}} \cdots \widetilde{\nabla}_{X_{p}} \widetilde{R}\right)(Y, Z)\right) & =\frac{c}{u^{q-1}} \sum_{i=1}^{p}\left(\nabla_{X_{1}} \cdots \nabla_{X_{p}} R\right)(Y, Z) X_{i},
\end{aligned}
$$

where $X_{i}, Y, Z \in T_{p} M$ and $c$ is a nonzero constant.

A first description of the holonomy of $(\widetilde{M}, \widetilde{g})$ was obtained in [21]. This description is the first part of the following proposition.

Proposition 4.2 ([21, Theorem 4.2]). Let $g$ be a semi-Riemannian metric on $M$ with holonomy algebra hol $(g)$ and $\tilde{g}$ the metric $\tilde{g}=2 \mathrm{~d} u \mathrm{~d} v+u^{2} g$ on $\mathbb{R}^{+} \times \mathbb{R} \times M$. Then

$$
\mathfrak{h o r}(\widetilde{g}) \subset \mathfrak{h o l}(g) \ltimes \mathbb{R}^{t, s} \subset \mathfrak{s o}(t, s) \ltimes \mathbb{R}^{t, s}=\mathfrak{s o}(t+1, s+1) \partial_{v},
$$

and

$$
\operatorname{pr}_{\mathfrak{s o}(t, s)}(\mathfrak{h o l}(\widetilde{g}))=\operatorname{hol}(g) .
$$

Moreover, if $(M, g)$ admits a nonzero parallel vector field $X$, then

$$
\mathfrak{h o r}(\widetilde{g}) \subset \mathfrak{h o l}(g) \ltimes X^{\perp},
$$

where $X^{\perp} \subset T_{p} M$ denotes the subspace orthogonal to $X_{p}$ with respect to $g$.

Proof. The proof of the first part of the proposition was given in [21] and uses equations (4.2) to compute explicitly the parallel transport in $(\widetilde{M}, \widetilde{g})$. Indeed, let $\widetilde{\gamma}:\left[t_{0}, t_{1}\right] \rightarrow \widetilde{M}$ be a piecewise smooth curve given by $\widetilde{\gamma}(t)=(u(t), v(t), \gamma(t))$ with a curve $\gamma$ in $M$. Let $Y(t)$ be 
a parallel vector field along $\gamma$ with respect to $\nabla$ and tangential to $M$. Then one checks that the vector field

$$
\tilde{Y}(t)=\frac{1}{u(t)} Y(t)+f(t) \cdot \partial_{v}
$$

is parallel with respect to $\tilde{\nabla}$ along $\tilde{\gamma}$, where $f(t)=\int_{t_{0}}^{t} g_{\gamma(s)}(\dot{\gamma}(s), Y(s)) \mathrm{d} s$. In particular, the parallel transport of $Z \in T_{\left(u\left(t_{0}\right), v\left(t_{0}\right), \gamma\left(t_{0}\right)\right)} M$ along $\widetilde{\gamma}$ is given by

$$
\widetilde{\mathcal{P}}_{\widetilde{\gamma}}(Z)=\frac{1}{u\left(t_{1}\right)} \mathcal{P}_{\gamma}(Z)+\left.\left(\int_{t_{0}}^{t_{1}} g_{\gamma(t)}\left(\dot{\gamma}(t),\left.\mathcal{P}_{\gamma}\right|_{\left[t_{0}, t\right]}(Z)\right) \mathrm{d} t\right) \partial_{v}\right|_{\tilde{\gamma}\left(t_{1}\right)} .
$$

This implies that for a loop $\widetilde{\gamma}$ starting and ending at $(u, v, p) \in \widetilde{M}$ we have that

$$
\operatorname{pr}_{\mathfrak{s o}(t, s)}\left(\widetilde{\mathcal{P}}_{\widetilde{\gamma}}\right)=\frac{1}{u} \mathcal{P}_{\gamma},
$$

which shows that $\operatorname{pr}_{\mathfrak{s o}(t, s)}(\mathfrak{h o r}(\widetilde{g}))=\mathfrak{h o r}(g)$.

For the second part, in the case where $(M, g)$ admits a parallel vector field $X$, the statement follows from the the Ambrose-Singer Holonomy Theorem and the second equation in (4.9) as $\left(\nabla_{X_{1}} \cdots \nabla_{X_{p}} R\right)\left(Y, Z, X_{i}, X\right)=0$ for all $X_{i} \in T M$ if $X$ is parallel.

Note that this does not establish the inclusion $\mathfrak{h o l}(g) \subset \mathfrak{h o l}(\widetilde{g})$. Hence, for a metric of the form $\widetilde{g}=2 \mathrm{~d} u \mathrm{~d} v+u^{2} g$ this result allows for the possibility that $\mathfrak{h o l}(\widetilde{g})$ is not completely determined by $\mathfrak{h o l}(g)$. Indeed, for the space of translations in $\mathfrak{h o l}(\widetilde{g})$,

$$
T:=\mathfrak{h o r}(\widetilde{g}) \cap \mathbb{R}^{t, s}
$$

we have the following possibilities:

(1) $T=\mathbb{R}^{t, s}$ : In this case the holonomy of $\widetilde{g}$ is completely determined by the holonomy of $g$ and we have $\mathfrak{h o l}(\widetilde{g})=\mathfrak{h o l}(g) \ltimes \mathbb{R}^{t, s}$.

(2) $T \neq \mathbb{R}^{t, s}$ : In this case we can distinguish two situations:

(a) $\mathfrak{h o l}(g) \subset \mathfrak{h o l}(\widetilde{g})$ : In this case there is a subspace of translations $T \subsetneq \mathbb{R}^{t, s}$ such that $\mathfrak{h o l}(\widetilde{g})=\mathfrak{h o l}(g) \ltimes T$.

(b) $\mathfrak{h o r}(g) \notin \mathfrak{h o r}(\widetilde{g})$.

In both cases in $(2)$ it seems as if $\mathfrak{h o l}(g)$ does not determine $\mathfrak{h o l}(\widetilde{g})$ completely and that further knowledge about the geometry of $g$ is needed in order to decide whether (a) or (b) occur, to determine $T$, etc. In Sections 5 and 6 we will study these questions further, first purely algebraically and then using geometric properties of $\widetilde{g}$. But first we will give some examples.

\subsection{Locally symmetric spaces and other examples.}

4.2.1. Locally symmetric spaces. Here we consider manifolds $(\widetilde{M}, \widetilde{g})$ that arise via the construction (4.1) from semi-Riemannian locally symmetric spaces $(M, g)$.

Theorem 4.3. Let $(M, g)$ be a semi-Riemannian locally symmetric space, i.e., a semiRiemannian manifold with $\nabla R=0$. For $(M, g)$ we consider the metric $\widetilde{g}=2 \mathrm{~d} u \mathrm{~d} v+u^{2} g$ on $\widetilde{M}=\mathbb{R}^{+} \times \mathbb{R} \times M$. Then

$$
\mathfrak{h o l}_{\widetilde{p}}(\widetilde{M}, \widetilde{g})=\mathfrak{h o l}_{p}(M, g) \ltimes T,
$$

where $T=\mathfrak{h o l}_{p}(M, g) V$ with $V=T_{p} M$ and $\widetilde{p}=(1,0, p) \in \widetilde{M}$. 
Proof. As a consequence of the Ambrose-Singer theorem and $\nabla R=0$ we have that

$$
\mathfrak{h o l}_{p}(M, g)=\operatorname{span}\left\{\left.R(X, Y)\right|_{p} \mid X, Y \in T_{p} M\right\} .
$$

The curvature $\widetilde{R}$ of $(\widetilde{M}, \widetilde{g})$ satisfies equation (4.3), which, together with equation (4.10), shows that $\mathfrak{g}=\mathfrak{h o l}(M, g)$ is contained in $\widetilde{\mathfrak{g}}=\mathfrak{h o l}_{p}(\widetilde{M}, \widetilde{g})$. Moreover, by Proposition 4.1 we have that

$$
\left(\widetilde{\nabla}_{\partial_{u}} \cdots \widetilde{\nabla}_{\partial_{u}} \widetilde{\nabla}_{X_{1}} \widetilde{R}\right)(X, Y) Z=\frac{c}{u^{q-1}} R\left(X, Y, Z, X_{1}\right) \partial_{v},
$$

for nonzero constant $c$ and $X, Y, Z, X_{1} \in T M$, and all other derivatives of $\widetilde{R}$ are zero. This implies the claim.

Corollary 4.4. Let $(M, g)$ be a semi-Riemannian locally symmetric space, which is locally the product of (non-flat) irreducible symmetric spaces. Then

$$
\mathfrak{h o l}_{\widetilde{p}}(\widetilde{M}, \widetilde{g})=\mathfrak{h o l}_{p}(M, g) \ltimes T_{p} M .
$$

Example 4.5. The following example shows that Corollary 4.4 does not extend to indecomposable symmetric spaces such as the Cahen-Wallach space of dimension $n=m+2$,

$$
(M, g)=\left(\mathbb{R}^{n}, g_{C W}=2 \mathrm{~d} x \mathrm{~d} z+\sum_{i, j=1}^{m} \lambda_{i j} y^{i} y^{j} \mathrm{~d} z^{2}+\sum_{i=1}^{m}\left(\mathrm{~d} y^{i}\right)^{2}\right),
$$

where $\left(x, y^{1}, \ldots, y^{m}, z\right)$ are global coordinates on $\mathbb{R}^{m+2}$ and where $S=\left(\lambda_{i j}\right)$ is a constant symmetric matrix with $\operatorname{det}(S) \neq 0$. In this case we have $\mathfrak{h o l}(M, g)=\mathbb{R}^{m} \subset \mathfrak{s o}(1, m+1)_{\partial_{x}}=$ $\mathfrak{s o}(m) \ltimes \mathbb{R}^{m}$ and $T=\operatorname{span}\left(\partial_{x}, \partial_{1} \ldots, \partial_{m}\right)$ where $\partial_{i}=\frac{\partial}{\partial y^{2}}$. We will explain these Lie algebras in more detail later on.

4.2.2. pp-waves and plane waves. The pp-waves are Lorentzian manifolds that are generalisations of Cahen-Wallach spaces. Again we consider $M=\mathbb{R}^{n}=\mathbb{R}^{m+2}$ with global coordinates $\left(x, y^{1}, \ldots, y^{m}, z\right)$ and $f$ a function $f=f\left(y^{1}, \ldots, y^{m}, z\right)$ of $y^{1}, \ldots, y^{m}$ and $z$ but not of $x$. Then a general pp-wave metric on $\mathbb{R}^{m+2}$ is given by

$$
g=2 \mathrm{~d} x \mathrm{~d} z+2 f\left(y^{1}, \ldots, y^{m}, z\right) \mathrm{d} z^{2}+\sum_{i=1}^{m}\left(\mathrm{~d} y^{i}\right)^{2} .
$$

The Levi-Civita connection and the curvature are determined by

$$
\nabla \partial_{x}=0, \quad \nabla_{\partial_{i}} \partial_{j}=0, \quad \nabla_{\partial_{z}} \partial_{i}=\partial_{i} f \partial_{x}, \quad \nabla_{\partial_{z}} \partial_{z}=\partial_{z} f \partial_{x}-\sum_{i=1}^{m} \partial_{i} f \partial_{i},
$$

and

$$
\left.\partial_{x}\right\lrcorner R=0, \quad R\left(\partial_{i}, \partial_{j}\right)=0, \quad R\left(\partial_{i}, \partial_{z}, \partial_{z}, \partial_{j}\right)=-\partial_{i} \partial_{j} f .
$$

In the basis $\left(\partial_{x}, \partial_{1}, \ldots, \partial_{m}, \partial_{z}-f \partial_{x}\right)$ the metric is given by

$$
\eta=\left(\begin{array}{ccc}
0 & 0 & 1 \\
0 & \mathbf{1}_{m} & 0 \\
1 & 0 & 0
\end{array}\right)
$$


and we can write the curvature and its derivatives as endomorphisms in $\mathfrak{s o}(\eta)$ as

$$
\left(\nabla_{X_{1}} \ldots \nabla_{X_{p}} R\right)\left(\partial_{i}, \partial_{z}\right)=\left(\begin{array}{ccc}
0 & \left(X_{1} \ldots X_{p} \partial_{i} \partial_{j}(f)\right)_{j=1}^{m} & 0 \\
0 & 0 & -\left(X_{1} \ldots X_{p} \partial_{i} \partial_{j}(f)\right)_{j=1}^{m} \\
0 & 0 & 0
\end{array}\right)
$$

where the $X_{i}$ are constant vector fields on $M=\mathbb{R}^{n}$. As for Cahen-Wallach spaces, their holonomy algebra contained in (and equal to, if the Hessian $\partial_{i} \partial_{j} f$ of $f$ is invertible) $\mathbb{R}^{m} \subset$ $\mathfrak{s o}(1, m+1) \partial_{x}$ and hence abelian.

Now we consider the semi-Riemannian manifold $(\widetilde{M}, \widetilde{g})$ of signature $(2, m+2)$ for a given pp-wave $(M, g)$ of dimension $n=m+2$. Then, by setting

$$
A_{q r k_{1} \ldots k_{s} i}:=\left(\widetilde{\nabla}_{\partial_{u}}^{q} \widetilde{\nabla}_{\partial_{z}}^{r} \widetilde{\nabla}_{\partial_{k_{1}}} \ldots \widetilde{\nabla}_{\partial_{k_{s}}} \widetilde{R}\right)\left(\partial_{i}, \partial_{z}\right)
$$

equations (4.9) in this case are

$$
\begin{aligned}
\operatorname{pr}_{\mathfrak{s o}(1, n-1)}\left(A_{q r k_{1} \ldots k_{s} i}\right) & =\frac{c}{u^{q}}\left(\begin{array}{ccc}
0 & \left(\partial_{k_{1}} \ldots \partial_{k_{s}} \partial_{i} \partial_{j} f^{(r)}\right)_{j=1}^{m} & 0 \\
0 & 0 & \vdots \\
0 & 0 & 0
\end{array}\right), \\
\operatorname{pr}_{\mathbb{R}^{1, n-1}}\left(A_{q r k_{1} \ldots k_{s} i}\right) & =\frac{c}{u^{q-1}}\left(s \partial_{k_{1}} \cdots \partial_{k_{s}} \partial_{i} f^{(r)} \partial_{x}+\sum_{j=1}^{m} \partial_{k_{1}} \cdots \partial_{k_{s}} \partial_{i} \partial_{j} f^{(r-1)} \partial_{j}\right) .
\end{aligned}
$$

where $f^{(r)}$ denotes the $r$-th derivative of $f$ with respect to the coordinate $z$. This shows that $\mathfrak{h o l}(\widetilde{M}, \widetilde{g}) \subset \mathfrak{h o l}(M, g) \ltimes \partial_{x}^{\perp}$, with $\partial^{\perp}=\operatorname{span}\left(\partial_{x}, \partial_{1}, \ldots, \partial_{m}\right)$, as claimed in Proposition 4.2. In general these projections could be coupled to each other, but for a special case we can say more:

Proposition 4.6. Let $(M, g)$ be a pp-wave as in (4.11) but with the condition that $f$ does not depend on $z$, i.e., $f=f\left(y^{1}, \ldots, y^{n}\right)$ and such that $\operatorname{det}\left(\partial_{i} \partial_{j} f\right) \neq 0$ at one point (or, more generally, such that at one point

$$
\operatorname{span}\left\{\mathrm{d}\left(\partial_{k_{1}} . . \partial_{k_{p}} f\right) \mid p \geqslant 1, k_{1}, \ldots, k_{p} \in \underline{m}\right\}=\left(\mathbb{R}^{m}\right)^{*},
$$

where $\underline{m}=\{1, \ldots, m\})$. Then

$$
\mathfrak{h o r}(\widetilde{M}, \widetilde{g})=\mathfrak{h o r}(M, g) \ltimes \partial_{x}^{\perp} .
$$

Proof. We evaluate formulae (4.9) for $r=1$ : since $f$ is independent of $z$, we have $f^{\prime}=0$ and hence

$$
\operatorname{pr}_{\mathfrak{s o}(1, m+1)}\left(\left(\widetilde{\nabla}_{\partial_{z}} \widetilde{\nabla}_{\partial_{k_{1}}} \cdots \widetilde{\nabla}_{\partial_{k_{p}}} \widetilde{R}\right)\left(\partial_{i}, \partial_{z}\right)\right)=0
$$

and

$$
\operatorname{pr}_{\mathbb{R}^{1, m+1}}\left(\left(\widetilde{\nabla}_{\partial_{z}} \widetilde{\nabla}_{\partial_{k_{1}}} \cdots \widetilde{\nabla}_{\partial_{k_{p}}} \widetilde{R}\right)\left(\partial_{i}, \partial_{z}\right)\right)=\sum_{j=1}^{m} \partial_{k_{1}} \cdots \partial_{k_{p}} \partial_{i} \partial_{j} f \partial_{j}
$$

If $\operatorname{det}\left(\partial_{i} \partial_{j} f\right) \neq 0$ (or if (4.14) holds at one point), this shows that $\operatorname{span}\left(\partial_{1} \ldots, \partial_{m}\right) \subset$ $\mathfrak{h o l}(\widetilde{M}, \widetilde{g}) \cap \mathbb{R}^{1, m+1}$. This space however is not invariant under $\mathfrak{h o l}(M, g)$ and is mapped under the adjoint representation in $\mathfrak{h o r}(\widetilde{M}, \widetilde{g})$ to $\mathbb{R} \partial_{x}$, so that $\mathfrak{h o r}(\widetilde{M}, \widetilde{g})=\mathfrak{h o l}(M, g) \ltimes \partial_{x}^{\perp}$. 
This proposition can be clearly generalised to functions $f$ that are polynomial, say of degree $d$, in $z$ (and have arbitrary dependence on the $y^{i}$ ). It suffices to replace $r=1$ in the proof with $r=d+1$ and the condition on $f$ by the corresponding condition on $f^{(d)}$. It does not hold however for general $f$ as the following example shows:

Example 4.7. Let $f(y, z)=\mathrm{e}^{z} y^{2}$ and $g$ a plane wave metrid on $\mathbb{R}^{3}$ defined by $f$,

$$
g=2 \mathrm{~d} x \mathrm{~d} z+\mathrm{e}^{z} y^{2} \mathrm{~d} z^{2}+\mathrm{d} y^{2} .
$$

Its curvature and derivatives thereof are given by equation (4.12) as follows

$$
\nabla_{\partial_{y}} R=0, \quad\left(\nabla_{\partial_{z}}^{(r)} R\right)\left(\partial_{y}, \partial_{z}\right)=2\left(\begin{array}{ccc}
0 & \mathrm{e}^{z} & 0 \\
0 & 0 & -\mathrm{e}^{z} \\
0 & 0 & 0
\end{array}\right)=: A(z),
$$

for all $r \geqslant 0$. Its holonomy algebra is one-dimensional. When we now consider the metric $\tilde{g}$, formula (4.13) shows that

$$
\begin{aligned}
\left(\widetilde{\nabla}_{\partial_{u}}^{q} \tilde{\nabla}_{\partial_{z}}^{r} \widetilde{R}\right)\left(\partial_{y}, \partial_{z}\right) & =\frac{c}{u^{q}}\left(\begin{array}{ccc}
0 & 2 u \mathrm{e}^{z} d y & 0 \\
0 & A(z) & -2 u \mathrm{e}^{z} \partial_{y} \\
0 & 0 & 0
\end{array}\right), \\
\left(\widetilde{\nabla}_{\partial_{u}}^{q} \tilde{\nabla}_{\partial_{z}}^{r} \widetilde{\nabla}_{\partial_{y}} \widetilde{R}\right)\left(\partial_{y}, \partial_{z}\right) & =\frac{2 c}{u^{q-1}} \mathrm{e}^{z} \partial_{x},
\end{aligned}
$$

with all other derivatives of the curvature being zero. Since $\widetilde{g}$ is analytic, its holonomy is determined by the derivatives of the curvature at a point, say at $v=x=y=z=$ 0 and $u=1$, and is spanned by the two matrices arising from $\left(\widetilde{\nabla}_{\partial_{u}}^{q} \widetilde{\nabla}_{\partial_{z}}^{r} \widetilde{R}\right)\left(\partial_{y}, \partial_{z}\right)$ and $\left(\widetilde{\nabla}_{\partial_{u}}^{q} \widetilde{\nabla}_{\partial_{z}}^{r} \widetilde{\nabla}_{\partial_{y}} \widetilde{R}\right)\left(\partial_{y}, \partial_{z}\right)$

$$
\left(\begin{array}{ccccc}
0 & 0 & 1 & 0 & 0 \\
0 & 0 & 1 & 0 & 0 \\
0 & 0 & 0 & -1 & -1 \\
0 & 0 & 0 & 0 & 0 \\
0 & 0 & 0 & 0 & 0
\end{array}\right), \quad\left(\begin{array}{ccccc}
0 & 0 & 0 & 1 & 0 \\
0 & 0 & 0 & 0 & -1 \\
0 & 0 & 0 & 0 & 0 \\
0 & 0 & 0 & 0 & 0 \\
0 & 0 & 0 & 0 & 0
\end{array}\right)
$$

This shows that the holonomy of $\widetilde{g}$ is abelian and is neither purely translational nor a semidirect sum of $\mathfrak{h o l}(g)$ with a Lie algebra of translations.

4.3. Lift of parallel objects. In this section we analyse how parallel objects on $(M, g)$, such as vector fields and vector distributions, lift to $(\widetilde{M}, \widetilde{g})$. First we analyse how certain vector fields on $M$ lift to $\widetilde{M}$.

Lemma 4.8. Let $\xi$ be a homothetic gradient vector field on $(M, g)$, i.e., a vector field with

$$
\nabla \xi=a \mathrm{Id}
$$

for a constant $a \in \mathbb{R}$ and such that $\xi^{b}$ is not only closed but exact, $\xi^{b}=d f$ for a smooth function $f$. Then the vector field $\tilde{\xi}$ defined by

$$
\tilde{\xi}=f \partial_{v}+\frac{1}{u} \xi-a \partial_{u}
$$

\footnotetext{
${ }^{2}$ Plane waves are pp-waves for which the function $f$ is a quadratic polynomial in the $y^{i}$ 's with $z$-dependent coefficients, i.e., $f\left(y^{1}, \ldots y^{m}, z\right)=\sum_{i, j=1}^{m} f_{i j}(z) y^{i} y^{j}$, with $\left(f_{i j}(z)\right)$ a symmetric matrix of functions of $z \in \mathbb{R}$.
} 
is parallel for $\widetilde{\nabla}$. In particular, if $\xi$ is parallel for $(M, g)$, then $\widetilde{\xi}=f \partial_{v}+\frac{1}{u} \xi$ is parallel for $(\widetilde{M}, \widetilde{g})$.

Proof. First note that the condition (4.15) implies that $\xi^{b}$ is closed, i.e., locally we can always find a function $f$ such that $\xi^{b}=d f$. Then we compute

$$
\widetilde{\nabla}_{\partial_{u}} \tilde{\xi}=-\frac{1}{u^{2}} \xi+\frac{1}{u} \widetilde{\nabla}_{\partial_{u}} \xi=0
$$

because of (4.2). Moreover, we have for every $X \in T M$ that

$$
\widetilde{\nabla}_{X} \widetilde{\xi}=d f(X) \partial_{v}+\frac{a}{u} X-g(\xi, X) \partial_{v}-a \widetilde{\nabla}_{X} \partial_{u}=0,
$$

again by (4.2) and $d f=\xi^{b}$.

In a similar way we can prove:

Lemma 4.9. Let $\mathbf{L}$ be a parallel null line bundle on $(M, g)$. Then the totally null 2-plane bundle $\mathbf{P}$ on $(\widetilde{M}, \widetilde{g})$ spanned by $\partial_{v}$ and $\mathbf{L}$ is parallel for $\widetilde{\nabla}$.

Proof. This follows from applying equation (4.2) to a recurrent null vector field $\xi$ spanning L and $\partial_{v}$ being parallel for $\widetilde{\nabla}$.

The following proposition will be used in Section 6 for the proof of Theorem 1.3.

Proposition 4.10. Let $(M, g)$ be a manifold with parallel null line bundle $\mathbf{L}$. Assume that the metric $\widetilde{g}=2 \mathrm{~d} u \mathrm{~d} v+u^{2} g$ admits a recurrent vector field in the span of $\partial_{v}$ and $\mathbf{L}$ that is not a multiple of $\partial_{v}$. Then locally $g$ admits a parallel null vector field in $\mathbf{L}$.

Proof. By Lemma 2.9 we can assume that $\mathbf{L}$ is spanned by a recurrent gradient vector field $\xi=\operatorname{grad}(f)$, i.e., with $\xi^{b}=d f$ and $\nabla \xi=\theta \otimes \xi$ with $\theta$ a multiple of $\xi^{b}$. Then the vector field

$$
\widetilde{\xi}=f \partial_{v}+\frac{1}{u} \xi
$$

satisfies

$$
\begin{aligned}
& \tilde{\nabla}_{\partial_{u}} \tilde{\xi}=0, \\
& \widetilde{\nabla}_{X} \widetilde{\xi}=\frac{1}{u} \theta(X) \xi=\theta(X)\left(\widetilde{\xi}-f \partial_{v}\right), \quad \text { for } X \in T M .
\end{aligned}
$$

Without loss of generality, the assumption implies that $\widetilde{g}$ admits a recurrent vector field of the form $\zeta=\widetilde{\xi}+h \partial_{v}$ for a function $h$. It defines a one-form $\alpha$ by $\widetilde{\nabla} \zeta=\alpha \otimes \zeta$. Then the fact that $\partial_{v}$ is parallel and equation (4.16) immediately show that

$$
\partial_{u} h=\alpha\left(\partial_{u}\right)=\partial_{v} h=\alpha\left(\partial_{v}\right)=0 .
$$

Equation (4.17) implies that

$$
\widetilde{\nabla}_{X} \zeta=\theta(X) \tilde{\xi}+(d h(X)-f \theta(X)) \partial_{v} .
$$

Hence the equation $\widetilde{\nabla} \zeta=\alpha \otimes \zeta$ implies that $\alpha=\theta$ and

$$
\mathrm{d} h=(f+h) \theta .
$$

Differentiating this and taking into account that $\mathrm{d} f \wedge \theta=\mathrm{d} h \wedge \theta=0$ gives

$$
0=(f+h) \mathrm{d} \theta
$$


If $\mathrm{d} \theta \neq 0$ this implies $h=-f$. This contradicts the above $\mathrm{d} h=(f+h) \theta$, as it would imply that $h$ and hence $f$ are constant. So we must have $\mathrm{d} \theta=0$. This however implies that one can rescale $\xi$ to a parallel null vector field.

Finally, for parallel distributions of $(M, g)$ we get

Lemma 4.11. Let $W \subset T M$ be a parallel distribution on $(M, g)$. Then the distribution $\mathbb{R} \partial_{v} \oplus W \subset T \widetilde{M}$ is parallel.

Proof. The distribution $W$ is locally spanned by vector fields $W_{1}, \ldots, W_{k}$. Then one checks that for $\widetilde{W}_{i}:=\partial_{v}+\frac{1}{u} W_{i}$ we have $\widetilde{\nabla}_{\partial_{u}} \widetilde{W}_{i}=0$ and

$$
\tilde{\nabla}_{X} \widetilde{W}_{i}=-g\left(X, W_{i}\right) \partial_{v}+\frac{1}{u} \nabla_{X} W_{i} \in \mathbb{R} \partial_{v} \oplus W
$$

for all $X \in T M$.

\section{Results about indecomposable subalgebras of $\mathfrak{s o}(t+1, s+1)$}

In this section we will prove several algebraic results about indecomposable subalgebras of $\mathfrak{s o}(t+1, s+1)$ stabilising a null line or a null vector. We will use these results in the next section when studying further the holonomy of metrics of the form $\widetilde{g}=2 \mathrm{~d} u \mathrm{~d} v+u^{2} g$.

5.1. Indecomposable subalgebras stabilising a null vector. In this section we will fix some notations and observe some fundamental facts about indecomposable subalgebras of $\mathfrak{s o}(t+1, s+1)$ stabilising a null vector. In particular, in this section we will see why the vector space $Z^{1}(\mathfrak{g}, V)$ of 1 -cocycles of a Lie algebra $\mathfrak{g}$ with values in a $\mathfrak{g}$-module $V$ comes into play. Recall that

$$
Z^{1}(\mathfrak{g}, V):=\left\{\varphi: \mathfrak{g}^{*} \otimes V \mid \varphi([X, Y])=X \varphi(Y)-Y \varphi(X) \text { for all } X, Y \in \mathfrak{g}\right\}
$$

and

where

$$
H^{1}(\mathfrak{g}, V):=\frac{Z^{1}(\mathfrak{g}, V)}{\mathrm{d} V}
$$

$$
\mathrm{d}: V \rightarrow Z^{1}(\mathfrak{g}, V), \quad \mathrm{d} v(X):=X v, \quad v \in V, \quad X \in \mathfrak{g} .
$$

Let $\tilde{V}$ be a semi-Euclidean vector space of signature $(t+1, s+1)$ with metric $\widetilde{g}$ and let $\mathbf{e}_{ \pm}$ be two null vectors such that $\widetilde{g}\left(\mathbf{e}_{-}, \mathbf{e}_{+}\right)=1$. We split $\widetilde{V}=L_{-} \oplus V \oplus L_{+}$with $L_{ \pm}=\mathbb{R} \cdot \mathbf{e}_{ \pm}$ and $V=\left(L_{-} \oplus L_{+}\right)^{\perp}$ which is equipped with the metric $g=\left.\widetilde{g}\right|_{V \times V}$. With respect to this splitting the stabiliser of $L_{-}$in $\mathfrak{s o}(\tilde{V})$, denoted by $\mathfrak{s o}(\tilde{V})_{L_{-}}$is given as

$$
\begin{aligned}
\mathfrak{s o}(\tilde{V})_{L_{-}} & =(\mathbb{R} \oplus \mathfrak{s o}(V)) \ltimes V \\
& :=\left\{(a, X, v):=\left(\begin{array}{ccc}
a & -v^{b} & 0 \\
0 & X & v \\
0 & 0 & -a
\end{array}\right) \mid a \in \mathbb{R}, X \in \mathfrak{s o}(V), v \in V\right\} .
\end{aligned}
$$

The action of $\mathfrak{s o}(\tilde{V})_{L_{-}}$on $\tilde{V}=L_{-} \oplus V \oplus L_{+} \cong \mathbb{R} \oplus V \oplus \mathbb{R}$ is given by

$$
(a, X, v) \cdot\left(\begin{array}{c}
r \\
u \\
s
\end{array}\right)=\left(\begin{array}{c}
a r-g(v, u) \\
X u+s v \\
-a s
\end{array}\right) \text {. }
$$


Furthermore we record the formula for the Lie bracket in $\mathfrak{s o}(\widetilde{V})_{L_{-}}$:

$$
[(a, X, v),(b, Y, w)]=(0,[X, Y],(X+a) w-(Y+b) v) .
$$

The stabiliser of the vector $\mathbf{e}_{-}$is given as $\mathfrak{s o}(\tilde{V})_{\mathbf{e}_{-}}=\mathfrak{s o}(V) \ltimes V$, i.e., is obtained by requiring $a$ to be zero in the above formulae. Note that, the adjoint action of $\mathfrak{s o}(\tilde{V}) \mathbf{e}_{-}=$ $\mathfrak{s o}(V) \ltimes V$ preserves the ideal $V$, whereas the linear action on $\widetilde{V}$ does not preserve the subspace $V \subset \tilde{V}$.

Furthermore note that there are natural projections $\operatorname{pr}_{V}$ and $\operatorname{pr}_{\mathfrak{s o}(V)}$ on $V$ and $\mathfrak{s o}(V)$. For a subalgebra $\tilde{\mathfrak{g}} \subset \mathfrak{s o}(V) \ltimes V$ we call $\mathfrak{g}:=\operatorname{pr}_{\mathfrak{s o}(V)}(\widetilde{\mathfrak{g}})$ the linear part of $\widetilde{\mathfrak{g}}$ and $T:=\tilde{\mathfrak{g}} \cap V$ the translations in $\tilde{\mathfrak{g}}$. Note that $\tilde{\mathfrak{g}} \subset \mathfrak{g} \ltimes V$ but in general $\mathfrak{g} \notin \tilde{\mathfrak{g}}$.

Proposition 5.1. Let $\tilde{\mathfrak{g}} \subset \mathfrak{s o}(\tilde{V})_{\mathbf{e}_{-}}=\mathfrak{s o}(V) \ltimes V$ be a subalgebra, $\mathfrak{g}$ its linear part and $T$ the translations in $\tilde{\mathfrak{g}}$. Then

(1) $T$ is an ideal in $\tilde{\mathfrak{g}}$.

(2) $T \subset V$ is invariant under $\mathfrak{g}$, and consequently $\mathfrak{g}$ acts on $V / T$.

(3) We have an inclusion of Lie algebras $\mathfrak{\mathfrak { g }} / T \subset \mathfrak{g} \ltimes V / T$.

(4) There is a $\varphi \in Z^{1}(\mathfrak{g}, V / T)$ such that $\mathfrak{\mathfrak { g }} / T=\{(X, \varphi(X)) \mid X \in \mathfrak{g}\}$.

(5) If $T$ has a $\mathfrak{g}$-invariant complement $T^{\prime}$, then there is a $\varphi \in Z^{1}\left(\mathfrak{g}, T^{\prime}\right)$, such that

$$
\tilde{\mathfrak{g}}=\mathfrak{h}_{\varphi} \ltimes T, \quad \text { where } \mathfrak{h}_{\varphi}=\{(X, \varphi(X)) \in \tilde{\mathfrak{g}} \mid X \in \mathfrak{g}\}
$$

Proof. Items 1 to 3 are obvious from the definitions. For Item 4 we define $\varphi(X)=v \bmod T$ if $(X, v) \in \tilde{\mathfrak{g}}$. Since $(X, v) \in \tilde{\mathfrak{g}}$ and $(X, w) \in \tilde{\mathfrak{g}}$ implies that $v-w \in T$, this map is well defined. From equation (5.3) we see that $\varphi$ is an element in $Z^{1}(\mathfrak{g}, V / T)$. Finally, Item 5 follows easily from Item 4 using the identification $V / T=T^{\prime}$ as $\mathfrak{g}$-modules.

Theorem 5.2. Let $\tilde{\mathfrak{g}} \subset \mathfrak{s o}(\tilde{V})_{\mathbf{e}_{-}}=\mathfrak{s o}(V) \ltimes V$ be a subalgebra acting indecomposably on $\tilde{V}$. Let $\mathfrak{g} \subset \mathfrak{s o}(V)$ and $T \subset V$ be respectively the linear part and translational ideal of $\tilde{\mathfrak{g}}$.

(1) If $T$ has a $\mathfrak{g}$-invariant complement $T^{\prime}$ and $H^{1}(\mathfrak{g}, V)=0$, then, up to conjugation in $\mathfrak{s o}(V) \ltimes V, \widetilde{\mathfrak{g}}=\mathfrak{g} \ltimes T$ and $T^{\perp}$ is degenerate or zero. In particular, if $T$ is nondegenerate and $H^{1}(\mathfrak{g}, V)=0$, then $T=V$.

(2) If $T$ is degenerate such that $L=T \cap T^{\perp}$ is a null line (this is the case for example when $T$ is degenerate and $g$ Lorentzian) and if the representation of $\mathfrak{g}$ on $V / L^{\perp}$ satisfies that $H^{1}\left(\mathfrak{g}, V / L^{\perp}\right)=0$, then $\mathfrak{g}$ acts trivially on $L$ or, up to conjugation in $\mathfrak{s o}(V) \ltimes V, \tilde{\mathfrak{g}}$ preserves $L$.

Proof. (1) First assume $V=T \oplus T^{\prime}$ is a $\mathfrak{g}$-invariant decomposition. In virtue of Proposition 5.1 $\tilde{\mathfrak{g}}=\mathfrak{h}_{\varphi} \ltimes T$, for some $\varphi \in Z^{1}\left(\mathfrak{g}, T^{\prime}\right)$. Since $Z^{1}(\mathfrak{g}, V)=\mathrm{d} V$ and $Z^{1}\left(\mathfrak{g}, T^{\prime}\right) \subset Z^{1}(\mathfrak{g}, V)$, we find a $v \in V$ such that

$$
\varphi(X)=X v
$$

for all $X \in \mathfrak{g}$. Then every element $(X, \varphi(X))=(X, X v) \in \mathfrak{h}_{\varphi}$ can be conjugated to $X$ by a conjugation with the translation given by $v$, i.e., with

$$
A_{v}=\left(\begin{array}{ccc}
1 & -v^{b} & -\frac{1}{2} g(v, v) \\
0 & \mathbf{1} & v \\
0 & 0 & 1
\end{array}\right) .
$$


Indeed, for each $X \in \mathfrak{g}$ we get

$$
A_{v}\left(\begin{array}{ccc}
0 & -(X v)^{b} & 0 \\
0 & X & X v \\
0 & 0 & 0
\end{array}\right) A_{v}^{-1}=\left(\begin{array}{ccc}
0 & 0 & 0 \\
0 & X & X v \\
0 & 0 & 0
\end{array}\right) A_{-v}=\left(\begin{array}{ccc}
0 & 0 & 0 \\
0 & X & 0 \\
0 & 0 & 0
\end{array}\right),
$$

using that $X \in \mathfrak{s o}(V)$. This shows that after conjugation with a translation, we have that $\mathfrak{g} \subset \tilde{\mathfrak{g}}$. Hence $\tilde{\mathfrak{g}}=\mathfrak{g} \ltimes T$, where $T=\tilde{\mathfrak{g}} \cap V$. Note that this already implies that $T$ is nonzero, because otherwise $\tilde{\mathfrak{g}}=\mathfrak{g} \subset \mathfrak{s o}(V)$, which contradicts indecomposability. Since $T$ is $\mathfrak{g}$ invariant, also the orthogonal complement $T^{\perp}$ of $T$ in $V$ is $\mathfrak{g}$ invariant. Then equation (5.2) shows that $T^{\perp} \subset \tilde{V}$ is also invariant under the action of $T \subset \mathfrak{s o}(\tilde{V})$ on $\tilde{V}$ and therefore $T^{\perp}$ is $\tilde{\mathfrak{g}}$-invariant. Hence, by indecomposability of $\tilde{\mathfrak{g}}, T^{\perp}$ has to be degenerate or zero.

(2) Assume that $T$ is degenerate such that $L:=T \cap T^{\perp}$ is a null line. By Item 2 of Proposition 5.1, $L$ is invariant under $\mathfrak{g}$. Moreover, by Item 5 of Proposition 5.1 we have that there is a $\varphi \in Z^{1}(\mathfrak{g}, V / T)$ such that $\tilde{\mathfrak{g}} / T=\{(X, \varphi(X)) \mid X \in \mathfrak{g}\} \subset \mathfrak{g} \ltimes V / T$. Hence, if $\widetilde{\varphi}: \mathfrak{g} \rightarrow V$ is a lift of $\varphi$ we can write $\tilde{\mathfrak{g}}=\mathfrak{h}_{\tilde{\varphi}}+T$, where $\mathfrak{h}_{\tilde{\varphi}}=\{(X, \widetilde{\varphi}(X)) \in \mathfrak{g} \ltimes V \mid X \in \mathfrak{g}\}$. Note that, since $T$ may not have an invariant complement, in general we do not have that $\tilde{\varphi} \in Z^{1}(\mathfrak{g}, V)$ and neither that $\mathfrak{h}_{\tilde{\varphi}}$ is a subalgebra.

Let $L^{\perp}$ be the hyperplane in $V$ that is orthogonal to $L$. It is $L \subset T \subset L^{\perp}$ and hence, by formula (5.2), $L$ is annihilated by the translations $T$ in $\tilde{\mathfrak{g}}=\mathfrak{h}_{\tilde{\varphi}}+T$. It remains to show that $L$ is invariant under $\mathfrak{h}_{\tilde{\varphi}}$, unless $\mathfrak{g}$ acts trivially on $L$. For this we consider the projection $\pi: V / T \rightarrow V / L^{\perp}$ and distinguish two cases:

Case 1: $\pi \circ \varphi: \mathfrak{g} \rightarrow V / L^{\perp}$ is zero. This means that the image of the lift $\tilde{\varphi}$ is contained in $L^{\perp}$. This however implies that $L$ is not only invariant under $\mathfrak{g}$ but also under $\tilde{\mathfrak{g}}=$ $\mathfrak{h}_{\tilde{\varphi}}+T$. Indeed, from formula (5.2) it follows for an element $(X, \widetilde{\varphi}(X)) \in \mathfrak{h}_{\tilde{\varphi}}$ and $\ell \in L$, that $(X, \widetilde{\varphi}(X)) \cdot \ell=X \cdot \ell-g(\widetilde{\varphi}(X), \ell) \mathrm{e}_{-}=X \cdot \ell \in L$, since $\widetilde{\varphi}(X) \in L^{\perp}$ and $\mathfrak{g}$ leaves $L$ invariant. Hence, in this case $L$ is $\tilde{\mathfrak{g}}$-invariant.

Case 2: $\pi \circ \varphi: \mathfrak{g} \rightarrow V / L^{\perp}$ is not zero, i.e., the image of $\widetilde{\varphi}$ is not contained in $L^{\perp}$. In this case, similarly to (1), we try to find a conjugation with a translation that shows that $L$ is invariant under $\mathfrak{h}_{\widetilde{\varphi}}$ (after conjugation). For $v \in V$ to be determined, we consider the associated translation $A_{v}$ as in equation (5.4). Then, as in (1), for an element

$$
(X, \tilde{\varphi}(X))=\left(\begin{array}{ccc}
0 & -(\widetilde{\varphi}(X))^{b} & 0 \\
0 & X & \widetilde{\varphi}(X) \\
0 & 0 & 0
\end{array}\right)
$$

we get that

$$
A_{v}(X, \widetilde{\varphi}(X)) A_{v}^{-1}=\left(\begin{array}{ccc}
0 & -(\widetilde{\varphi}(X)-X v)^{b} & 0 \\
0 & X & \widetilde{\varphi}(X)-X v \\
0 & 0 & 0
\end{array}\right) .
$$

Fix $\ell \in L$ and $\hat{\ell} \in V$ such that $g(\ell, \hat{\ell})=1$. Then define $0 \neq \lambda \in \mathfrak{g}^{*}$ and $\rho \in \mathfrak{g}^{*}$ by $\widetilde{\varphi}(X)=\lambda(X) \hat{\ell} \bmod L^{\perp}$ and $X \ell=-\rho(X) \ell$, for $X \in \mathfrak{g}$. This is summarised in $(X, \widetilde{\varphi}(X)) \cdot \ell=$ $-\lambda(X) \mathrm{e}_{-}-\rho(X) \ell$. It also implies that $X \hat{\ell}=\rho(X) \hat{\ell} \bmod L^{\perp}$, i.e., $\rho: \mathfrak{g} \rightarrow \mathfrak{g l}\left(V / L^{\perp}\right)$ is the induced representation of $\mathfrak{g}$ on $V / L^{\perp}$. If we assume that $\mathfrak{g}$ does not act trivially on $L, \rho$ is not zero. The key observation now is that $H^{1}\left(\mathfrak{g}, V / L^{\perp}\right)=0$ implies that $\lambda=c \rho$ for a constant $c$. Indeed, $\varphi \in Z^{1}(\mathfrak{g}, V / T)$ induces an element $\bar{\varphi} \in Z^{1}\left(\mathfrak{g}, V / L^{\perp}\right)$. So $H^{1}\left(\mathfrak{g}, V / L^{\perp}\right)=0$ implies 
that $\bar{\varphi}(X)=X\left(c \hat{\ell} \bmod L^{\perp}\right)=c X \hat{\ell} \bmod L^{\perp}=c \rho(X) \hat{\ell} \bmod L^{\perp}$ and thus $\tilde{\varphi}(X)=c \rho(X) \hat{\ell}$ $\bmod L^{\perp}$.

Now, in equation (5.4) we set $v:=c \hat{\ell}$. Taking into account that $g(\hat{\ell}, \ell)=1$, formula (5.5) shows that

$$
A_{v}(X, \widetilde{\varphi}(X)) A_{v}^{-1} \cdot \ell=-(\lambda(X)-c \rho(X)) \mathbf{e}_{-}-\rho(X) \ell=-\rho(X) \ell .
$$

This shows that after conjugation with a translation the null line $L$ is invariant under $\mathfrak{h}_{\tilde{\varphi}}$ and hence under $\tilde{\mathfrak{g}}$.

Example 5.3. Consider $\mathfrak{g}=\mathbb{R}^{n} \subset \mathfrak{s o}(n) \ltimes \mathbb{R}^{n}=\mathfrak{s o}(1, n+1)_{\mathrm{e}_{0}}$, where $\mathrm{e}_{0} \in \mathbb{R}^{1, n+1}$ is a null vector. Then for $T=\mathbb{R} \cdot \mathrm{e}_{0}$ one can check that $\tilde{\mathfrak{g}}=\mathfrak{g} \ltimes T \subset \mathfrak{s o}(2, n+2) \mathrm{e}_{-}$is indecomposable. Similarly, for $T=\operatorname{span}\left(\mathrm{e}_{0}, \ldots \mathrm{e}_{n}\right), \tilde{\mathfrak{g}}=\mathfrak{g} \ltimes T$ is indecomposable. Note that the latter is the holonomy algebra of a $(\widetilde{M}, \widetilde{g})$ for a Cahen-Wallach space $\left(M, g_{C W}\right)$ of dimension $n+2$ presented in Example 4.5.

5.2. Indecomposable subalgebras with completely reducible linear part. The main result of this section is Theorem 5.5. which is a generalisation to arbitrary signature of a result in [7] for an indecomposable stabiliser in $\mathfrak{s o}(1, n+1)$ of a null vectors. It gives a description of all indecomposable subalgebras $\tilde{\mathfrak{g}} \subset \mathfrak{s o}(\widetilde{V})_{\mathbf{e}_{-}}=\mathfrak{s o}(V) \ltimes V$ with completely reducible linear part and non-degenerate translational part.

The main results of this and the next section use a result about Lie algebra cohomology 4 , which we will present first. In the following, for a $\mathfrak{g}$-module $V$, we denote by $V^{\mathfrak{g}}$ the $\mathfrak{g}$ invariant vectors,

$$
V^{\mathfrak{g}}=\{v \in V \mid X v=0 \text { for all } X \in \mathfrak{g}\} .
$$

Theorem 5.4 ([19, Theorem 13], [28, Theorem 2.28]). Let $\mathfrak{g}$ be a Lie algebra and $V$ a $\mathfrak{g}$ module, both finite-dimensional and over a field $\mathbb{F}$ of characteristic zero. Assume that there is an ideal $\mathfrak{b}$ in $\mathfrak{g}$ such that

(1) there is a subalgebra $\mathfrak{h}$ in $\mathfrak{g}$ such that $\mathfrak{g}=\mathfrak{h} \ltimes \mathfrak{b}$. and

(2) $V$ and $\mathfrak{g}$ are completely reducible as $\mathfrak{h}$-modules.

Then

$$
H^{p}(\mathfrak{g}, V) \simeq \sum_{i+j=p} H^{i}(\mathfrak{h}, \mathbb{F}) \otimes H^{j}(\mathfrak{b}, V)^{\mathfrak{g}} .
$$

In particular, when $p=1$,

$$
H^{1}(\mathfrak{g}, V) \simeq H^{1}(\mathfrak{b}, V)^{\mathfrak{g}}+(\mathfrak{h} /[\mathfrak{h}, \mathfrak{h}])^{*} \otimes V^{\mathfrak{g}} .
$$

The original version of this theorem is due to Hochschild and Serre [19, Theorem 13], in which the existence of $\mathfrak{h}$ was not assumed but that $\mathfrak{g} / \mathfrak{b}$ is semisimple. Solleveld proved the generalisation that is given here in his Master's thesis [28, Theorem 2.28]. Equation (5.6) for $p=1$ follows from the facts that $H^{0}(\mathfrak{h}, \mathbb{F})=\mathbb{F}, H^{0}(\mathfrak{b}, V)=V^{\mathfrak{b}}$ and that $H^{1}(\mathfrak{h}, \mathbb{F})$ is isomorphic to $(\mathfrak{h} /[\mathfrak{h}, \mathfrak{h}])^{*}$.

Now we turn to the main result of this section. We use the same conventions as in Section 5.1 .

\footnotetext{
${ }^{3}$ We point out that in [7] a similar result for the stabiliser in $\mathfrak{s o}(1, n+1)$ of a null line is given.

${ }^{4}$ We do have self-contained proofs of Theorems 5.5] and 5.7 that do not use Theorem [5.4, but for the sake of brevity we do not present them here as they are longer.
} 
Theorem 5.5. Let $\tilde{\mathfrak{g}} \subset \mathfrak{s o}(\tilde{V}) \mathbf{e}_{-}=\mathfrak{s o}(V) \ltimes V$ an indecomposable subalgebra which satisfies the following properties

(1) $\mathfrak{g}=\operatorname{pr}_{\mathfrak{s o}(V)}(\mathfrak{\mathfrak { g }})$ acts completely reducibly on $V$, and

(2) the translational ideal $T=\tilde{\mathfrak{g}} \cap V$ is non-degenerate.

Under these assumptions, let $\mathfrak{g}=\mathfrak{z} \oplus \mathfrak{g}^{\prime}$ be the decomposition of $\mathfrak{g}$ into its centre and the semisimple derived Lie algebra. Then, $\mathfrak{g}$ acts trivially on $T^{\perp}$ and $T \neq 0$. Moreover, there is a linear map $\varphi: \mathfrak{g} \rightarrow T^{\perp}$ with $\left.\varphi\right|_{\mathfrak{g}^{\prime}}=0$ such that after conjugation in $\mathfrak{s o}(V) \ltimes V, \widetilde{\mathfrak{g}}$ is of the form $\tilde{\mathfrak{g}}=\mathfrak{h}_{\varphi} \ltimes T$, where

$$
\mathfrak{h}_{\varphi}=\{(X, \varphi(X)) \in \mathfrak{s o}(V) \ltimes V \mid X \in \mathfrak{g}\},
$$

and the image of $\varphi$ is co-null in $T^{\perp}$, i.e., $(\operatorname{im} \varphi)^{\perp} \subset T^{\perp}$ is totally null.

The proof of this theorem is based on a lemma which will follow from Theorem 5.4. Since $V$ is a completely reducible module, $\mathfrak{g}$ is reductive and hence $\mathfrak{g}=\mathfrak{z} \oplus \mathfrak{g}^{\prime}$, where $\mathfrak{g}^{\prime}=[\mathfrak{g}, \mathfrak{g}]$ is semisimple, $\mathfrak{z}$ is the centre of $\mathfrak{g}$ and we denote the projection to $\mathfrak{z}$ by $\pi_{\mathfrak{z}}: \mathfrak{g} \rightarrow \mathfrak{z}$.

Lemma 5.6. Let $V$ be a semi-Euclidean vector space and $\mathfrak{g} \subset \mathfrak{s o}(V)$ be a Lie subalgebra which acts completely reducibly on $V$. Then

$$
Z^{1}(\mathfrak{g}, V)=\mathrm{d} V \oplus \iota\left(Z^{1}\left(\mathfrak{z}, V^{\mathfrak{g}}\right)\right),
$$

where $\mathfrak{z}$ the center of $\mathfrak{g}$ and $\iota: Z^{1}\left(\mathfrak{z}, V^{\mathfrak{g}}\right) \rightarrow Z^{1}(\mathfrak{g}, V)$ is the inclusion $\iota(\varphi)=\varphi \circ \pi_{\mathfrak{z}}$ with $\pi_{\mathfrak{z}}: \mathfrak{g} \rightarrow \mathfrak{z}$. In particular,

$$
H^{1}(\mathfrak{g}, V) \simeq H^{1}\left(\mathfrak{z}, V^{\mathfrak{g}}\right) .
$$

Proof. First note that for $\varphi \in Z^{1}\left(\mathfrak{z}, V^{\mathfrak{g}}\right)=\operatorname{Hom}\left(\mathfrak{z}, V^{\mathfrak{g}}\right), \iota(\varphi)$ is indeed a cocycle in $Z^{1}(\mathfrak{g}, V)$. Moreover, with $V$ completely reducible we have $V^{\mathfrak{g}} \cap \mathfrak{g} V=\{0\}$ and hence that

$$
\mathrm{d} V \cap \iota\left(Z^{1}\left(\mathfrak{z}, V^{\mathfrak{g}}\right)\right)=\{0\} .
$$

It remains to show that

$$
H^{1}(\mathfrak{g}, V) \simeq Z^{1}\left(\mathfrak{z}, V^{\mathfrak{g}}\right) .
$$

But we can apply Theorem 5.4 to $\mathfrak{g}, \mathfrak{b}=\mathfrak{z}$ and $\mathfrak{h}=\mathfrak{g}^{\prime}$ to get from equation (5.6) that

$$
H^{1}(\mathfrak{g}, V) \simeq H^{1}(\mathfrak{z}, V)^{\mathfrak{g}} .
$$

Therefore it remains to show that $H^{1}(\mathfrak{z}, V)^{\mathfrak{g}}$ is isomorphic to $Z^{1}\left(\mathfrak{z}, V^{\mathfrak{g}}\right)$. We note that

$$
H^{1}(\mathfrak{z}, V)^{\mathfrak{g}}=\left\{[\varphi] \in H^{1}(\mathfrak{z}, V) \mid \varphi \in Z^{1}(\mathfrak{z}, V): \forall X \in \mathfrak{g} \exists v \in V: X \varphi=\mathrm{d}_{\mathfrak{z}} v\right\} .
$$

Clearly, $Z^{1}\left(\mathfrak{z}, V^{\mathfrak{g}}\right)$ injects into $H^{1}(\mathfrak{z}, V)^{\mathfrak{g}}$ by mapping a cocycle to its equivalence class in $H^{1}(\mathfrak{z}, V)^{\mathfrak{g}}$, but we have to show that this is surjective.

For this, note that if $[\varphi] \in H^{1}(\mathfrak{z}, V)^{\mathfrak{g}}$, we have that $\varphi \in Z^{1}(\mathfrak{z}, V)$ is such that for each $X \in \mathfrak{g}$, there is a $v_{X} \in V$ such that

$$
X \varphi(Z)=Z v_{X}
$$

This defines a linear map $\hat{\varphi}: \mathfrak{g} \rightarrow V / V^{\mathfrak{z}}$ by the relation

$$
\hat{\varphi}(X)=v_{X}+V^{\mathfrak{z}} .
$$

Since $Z \in \mathfrak{z}$, it is

$$
Z v_{[X, Y]}=[X, Y] \varphi(Z)=Z\left(X v_{Y}-Y v_{Y}\right)
$$


and so $\hat{\varphi}$ is a cocycle, i.e., $\hat{\varphi} \in Z^{1}\left(\mathfrak{g}, V / V^{\mathfrak{z}}\right)$. This induces a linear map

$$
\Psi: H^{1}(\mathfrak{z}, V)^{\mathfrak{g}} \ni[\varphi] \longmapsto[\hat{\varphi}] \in H^{1}\left(\mathfrak{g}, V / V^{\mathfrak{z}}\right),
$$

which clearly has the kernel $Z^{1}\left(\mathfrak{z}, V^{\mathfrak{g}}\right)$. Therefore

$$
H^{1}(\mathfrak{z}, V)^{\mathfrak{g}} / Z^{1}\left(\mathfrak{z}, V^{\mathfrak{g}}\right) \simeq \operatorname{im}(\Psi) \subset H^{1}\left(\mathfrak{g}, V / V^{\mathfrak{z}}\right) .
$$

Now we use again equation (5.6) in Theorem 5.4 to get that

$$
H^{1}\left(\mathfrak{g}, V / V^{\mathfrak{z}}\right) \simeq H^{1}\left(\mathfrak{z}, V / V^{\mathfrak{z}}\right)^{\mathfrak{g}} .
$$

The last step in the proof is to show that $H^{1}\left(\mathfrak{z}, V / V^{\mathfrak{z}}\right)=\{0\}$. For this we set $W:=V / V^{\mathfrak{z}}$ and we have to show that $Z^{1}(\mathfrak{z}, W)=\mathrm{d} W$. The $\mathfrak{z}$-module $W$ is an orthogonal sum of 2dimensional indecomposable modules $W_{i}$ and $Z^{1}(\mathfrak{z}, W)=\oplus_{i} Z^{1}\left(\mathfrak{z}, W_{i}\right)$. Therefore we can assume without loss of generality that $W=W_{1}$ is 2-dimensional. Let us denote by $I$ a generator of the 1-dimensional Lie algebra $\mathfrak{s o}(W)$ such that $I^{2}=\epsilon \mathrm{Id}, \epsilon= \pm 1$. Then there exists $0 \neq \lambda \in \mathfrak{z}^{*}$ such that $X v=\lambda(X) I v$ for all $X \in \mathfrak{z}$ and $v \in W$. Given $\varphi \in Z^{1}(\mathfrak{z}, W)$, we have

$$
0=X \varphi(Y)-Y \varphi(X)=\lambda(X) I \varphi(Y)-\lambda(Y) I \varphi(X),
$$

for all $X, Y \in \mathfrak{z}$. The latter equation implies that there exists a vector $v \in W$ such that

$$
I \varphi(X)=\lambda(X) v,
$$

for all $X \in \mathfrak{z}$. This shows that $\varphi=\epsilon \lambda \otimes I v=\epsilon \mathrm{d} v \in \mathrm{d} W$ and hence that $H^{1}\left(\mathfrak{z}, V / V^{\mathfrak{z}}\right)=\{0\}$.

This implies that $\operatorname{im}(\Psi)=\{0\}$ and hence that $Z^{1}\left(\mathfrak{z}, V^{\mathfrak{g}}\right)=H^{1}(\mathfrak{z}, V)^{\mathfrak{g}} \simeq H^{1}(\mathfrak{g}, V)$.

Now we are in a position to prove Theorem 5.5 .

Proof of Theorem 5.5. From Proposition 5.1 we have that $\tilde{\mathfrak{g}}=\mathfrak{h}_{\varphi} \ltimes T$, where $\mathfrak{h}_{\varphi}$ is given by equation (5.7) with $\varphi \in Z^{1}\left(\mathfrak{g}, T^{\perp}\right)$. It remains to verify that $\left.\varphi\right|_{\mathfrak{g}^{\prime}}=0$. Lemma 5.6] shows that, up to conjugation of $\mathfrak{g}$ in $\mathfrak{s o}(V) \ltimes V$ by a translation in $T^{\perp}$ we have $\varphi \in \iota\left(Z^{1}\left(\mathfrak{z}, T^{\perp} \cap V^{\mathfrak{g}}\right)\right)$. This shows that $\varphi$ vanishes on $\mathfrak{g}^{\prime}$ and takes values in $T^{\perp} \cap V^{\mathfrak{g}}$. The $\mathfrak{g}$-invariant decomposition

$$
T^{\perp}=\left(T^{\perp} \cap V^{\mathfrak{g}}\right) \stackrel{\perp}{\oplus} \mathfrak{g} T^{\perp}
$$

shows that the subspace $\mathfrak{g} T^{\perp} \subset V$ is non-degenerate. Let us check that it is not only invariant under $\mathfrak{g}$ but also under $\mathfrak{\mathfrak { g }}$. For this is suffices to observe that, by our description of $\tilde{\mathfrak{g}}$ and the fact that $\operatorname{im} \varphi \subset T^{\perp} \cap V^{\mathfrak{g}}$, the translational part of any element of $\tilde{\mathfrak{g}}$ is contained in $\left(T^{\perp} \cap V^{\mathfrak{g}}\right) \oplus T$. Therefore it is perpendicular to $\mathfrak{g} T^{\perp}$, which shows that $\mathfrak{g} T^{\perp} \subset V \subset \tilde{V}$ is $\tilde{\mathfrak{g}}$-invariant. Since $\tilde{\mathfrak{g}}$ is indecomposable this proves that $\mathfrak{g} T^{\perp}=0$.

Note that this implies that $T \neq 0$, because otherwise $T^{\perp}=V$ and hence $\mathfrak{g}=0$ and $\tilde{\mathfrak{g}}=T=0$, which contradicts the indecomposability of $\widetilde{\mathfrak{g}}$.

Finally, let $(\operatorname{im} \varphi)^{\perp}$ be the orthogonal space of $\operatorname{im} \varphi$ in $T^{\perp}$ and $W$ be a $\mathfrak{g}$-invariant complement of $\operatorname{im} \varphi \cap(\operatorname{im} \varphi)^{\perp}$ in $(\operatorname{im} \varphi)^{\perp}$. Then $W$ is non-degenerate. Again it is not only $\mathfrak{g}$-invariant but also $\widetilde{\mathfrak{g}}$-invariant because the translational part of any element in $\tilde{\mathfrak{g}}$ is contained in $(\operatorname{im} \varphi) \oplus T$ and $W \subset(\operatorname{im} \varphi)^{\perp} \subset T^{\perp}$. Since $\tilde{\mathfrak{g}}$ is indecomposable this shows that $W=0$ and, hence, that $(\operatorname{im} \varphi)^{\perp} \subset \operatorname{im} \varphi$. 
5.3. Cohomology of indecomposable subalgebras in $\mathfrak{s o}(1, n+1)$. In this section we compute the 1-cocycles for subalgebras $\mathfrak{g}$ of $\mathfrak{s o}(1, n+1)$ that act indecomposably on $V=$ $\mathbb{R}^{1, n+1}$. Such a subalgebra is either irreducible, in which case it is equal to $\mathfrak{s o}(1, n+1)$ 11 and hence $H^{1}(\mathfrak{g}, V)=0$, or admits a parallel null-line $L=L_{-}=\mathbb{R e}_{-}$. That such a subalgebra belongs to one of the four types discussed in the proof of Theorem 5.7 below, was proven in 7 .

In the following we will use equations (5.2) and (5.3) and the identifications in Section 5.1 with $(\tilde{V}, \tilde{g}, V, g)$ replaced by $\left(V, g, V_{0}, g_{0}\right)$. Note that $g_{0}=\left.g\right|_{V_{0} \times V_{0}}$ is the standard Euclidean scalar product on $V_{0}=\mathbb{R}^{n}$. We will use the standard decomposition $V=\mathbb{R} \cdot \mathrm{e}_{-} \oplus V_{0} \oplus \mathbb{R} \cdot \mathrm{e}_{+}$ and the notation $\mathfrak{g}_{0}=\operatorname{pr}_{\mathfrak{s o}\left(V_{0}\right)}(\mathfrak{g}), \mathfrak{g}_{0}^{\prime}=\left[\mathfrak{g}_{0}, \mathfrak{g}_{0}\right], \mathfrak{z}=\mathfrak{z}\left(\mathfrak{g}_{0}\right)$ for a subalgebra $\mathfrak{g} \subset \mathfrak{s o}(V)_{L}$.

Theorem 5.7. Let $V=\mathbb{R} \cdot \mathrm{e}_{-} \oplus V_{0} \oplus \mathbb{R} \cdot \mathrm{e}_{+}$be the Minkowski space with null vectors $\mathrm{e}_{ \pm}$and Euclidean vector space $V_{0}$, and let $\mathfrak{g} \subset \mathfrak{s o}(V)_{L} \subset \mathfrak{s o}(V)$ be an indecomposable subalgebra. Then

$$
H^{1}(\mathfrak{g}, V)=0
$$

or $\mathfrak{g}$ annihilates $\mathbf{e}_{-}$.

Proof. First note that if $\operatorname{dim}(V)=2$, i.e., $V_{0}=0$, then

$$
\mathfrak{g}=\mathfrak{s o}(1,1)=\mathbb{R}\left(\begin{array}{cc}
1 & 0 \\
0 & -1
\end{array}\right),
$$

and $H^{1}(\mathfrak{g}, V)$ clearly is trivial.

If $\operatorname{dim}(V) \geqslant 3$, then according to [7], any indecomposable subalgebra $\mathfrak{g}$ of $\mathfrak{s o}(V)_{L}$, belongs to one of four different types. Two of them annihilate $\mathrm{e}_{-}$, whereas the other two act nontrivially on $\mathbb{R} \cdot \mathrm{e}_{-}$. The latter are given as follows, where $\mathfrak{z}$ denotes the centre of $\mathfrak{g}_{0}=\mathfrak{z} \oplus \mathfrak{g}_{0}^{\prime}$ with $\mathfrak{g}_{0}^{\prime}=\left[\mathfrak{g}_{0}, \mathfrak{g}_{0}\right]$ semisimple:

(1) $\mathfrak{g}=\left(\mathbb{R} \oplus \mathfrak{g}_{0}\right) \ltimes V_{0}$. We can set

$$
\mathfrak{b}:=(\mathbb{R} \oplus \mathfrak{z}) \ltimes V_{0} .
$$

Then $\mathfrak{g} / \mathfrak{b}=\mathfrak{g}_{0}^{\prime}$ is semisimple and acts completely reducibly on $V=\mathbb{R e}_{-} \oplus V_{0} \oplus \mathbb{R e}_{+}$.

(2) $\mathfrak{g}=\left(\mathfrak{h}_{f} \oplus \mathfrak{g}_{0}^{\prime}\right) \ltimes V_{0}$, with $0 \neq f \in \mathfrak{z}^{*}$ and $\mathfrak{h}_{f}=\{(f(Z), Z) \mid Z \in \mathfrak{z}\} \subset \mathbb{R} \oplus \mathfrak{z}$. Here we set

$$
\mathfrak{b}:=\mathfrak{h}_{f} \ltimes V_{0},
$$

so that $\mathfrak{g} / \mathfrak{b}=\mathfrak{g}_{0}^{\prime}$ acts again completely reducibly on $V$.

Now we apply Theorem 5.4 to $\mathfrak{g}$, the ideal $\mathfrak{b}$ as given in the above and $\mathfrak{g}_{0}^{\prime}=\mathfrak{g} / \mathfrak{b}$. Since $\mathfrak{g}_{0}^{\prime}$ is semisimple, the second summand in (5.6) vanishes and we get

$$
H^{1}(\mathfrak{g}, V) \simeq H^{1}(\mathfrak{b}, V)^{\mathfrak{g}} .
$$

In order to determine $H^{1}(\mathfrak{b}, V)$ we can apply Theorem 5.4 again, this time to $\mathfrak{b}$, the ideal $\mathfrak{a}=V_{0}$ and the subalgebra $\mathfrak{h}=\mathbb{R} \oplus \mathfrak{z}$ in case (1) and $\mathfrak{h}=\mathfrak{h}_{f}$ in case (2). In both cases $\mathfrak{h}$ is abelian and acts completely reducibly on $\mathfrak{b}$ and on $V$, so the assumptions of Theorem 5.4 are satisfied and we get

$$
H^{1}(\mathfrak{b}, V) \simeq H^{1}(\mathfrak{a}, V)^{\mathfrak{b}}+\mathfrak{h}^{*} \otimes V^{\mathfrak{b}} .
$$

Since for both types of $\mathfrak{g}, \mathfrak{b}$ scales e_ and contains $\mathfrak{a}=V_{0}$, we have that $V^{\mathfrak{b}}=\{0\}$, cf. (5.2). So it remains to show that $\left(H^{1}(\mathfrak{a}, V)^{\mathfrak{b}}\right)^{\mathfrak{g}}=H^{1}(\mathfrak{a}, V)^{\mathfrak{g}}$ is trivial. Even though $\mathfrak{a}=V_{0}$ is abelian, we cannot apply Lemma [5.6 to find $H^{1}(\mathfrak{a}, V)$, because $\mathfrak{a}$ does not act completely reducibly on $V$. Instead, we first note that if $\operatorname{dim}(\mathfrak{a})=\operatorname{dim}\left(V_{0}\right)=1$, then $Z^{1}(\mathfrak{a}, V)=\mathfrak{a}^{*} \otimes V$, 
$\mathrm{d} V=\mathfrak{a}^{*} \otimes\left(\mathbb{R e}_{-} \oplus V_{0}\right)$ and the line $\mathfrak{a}^{*} \otimes \mathrm{e}_{+} \subset Z^{1}(\mathfrak{a}, V)$ projects isomorphically onto $H^{1}(\mathfrak{a}, V)$. From (5.2) we see that the action of an element $(a, X, v) \in \mathfrak{g}$ on $H^{1}(\mathfrak{a}, V)$ is given by multiplication with $-a$. Since for both types of $\mathfrak{g}$ there are elements with $a \neq 0$, we conclude that $H^{1}(\mathfrak{a}, V)^{\mathfrak{g}}=0$. Thus we can assume that $\operatorname{dim}\left(V_{0}\right) \geqslant 2$. Then $\varphi \in Z^{1}(\mathfrak{a}, V)$ splits into components $\varphi=\left(\varphi_{-}, \varphi_{0}, \varphi_{+}\right)$with respect to $V=\mathbb{R} \cdot \mathrm{e}_{-} \oplus V_{0} \oplus \mathbb{R} \cdot \mathrm{e}_{+}$and with $\varphi_{ \pm} \in \mathfrak{a}^{*}$ and $\varphi_{0} \in V_{0}^{*} \otimes V_{0}$. From (5.2) we see that $u \in V_{0}=\mathfrak{a}$ acts on $\left(v_{-}, v, v_{+}\right) \in V$ as

$$
u \cdot\left(v_{-}, v, v_{+}\right)=\left(-u^{\top} v, v_{+} u, 0\right) \text {. }
$$

Since $\mathfrak{a}$ is abelian, the cocycle condition for $\varphi$ yields

$$
u^{\top} \varphi_{0}(v)-v^{\top} \varphi_{0}(u)=0, \quad \varphi_{+}(u) v-\varphi_{+}(v) u=0,
$$

for all $u, v \in V_{0}$. Since $\operatorname{dim}\left(V_{0}\right) \geqslant 2$ the second equation implies that $\varphi_{+}=0$. The first equation implies that $\varphi_{0}$ is a symmetric endomorphism of $V_{0}$. This shows that $Z^{1}(\mathfrak{a}, V)=$ $V_{0}^{*} \oplus S\left(V_{0}\right)$ and that

$$
H^{1}(\mathfrak{a}, V) \simeq S_{0}\left(V_{0}\right),
$$

where $S\left(V_{0}\right)$ and $S_{0}\left(V_{0}\right)$ denote the symmetric and the symmetric trace-free endomorphisms of $V_{0}$. Hence, every element $[\varphi] \in H^{1}(\mathfrak{a}, V)^{\mathfrak{g}}$ can be represented by a symmetric trace free-matrix $S$. Therefore the equation that $[\varphi]$ is $\mathfrak{g}$-invariant, which means that for every $(a, X, v) \in \mathfrak{g}$ there is a $\left(w_{-}, w, w_{+}\right) \in V$ such that

$$
(a, X, v) \cdot \varphi=\mathrm{d}\left(w_{-}, w, w_{+}\right)
$$

becomes, by (5.2),

$$
(a, X, v)(\varphi(u))-\varphi([(a, X, v),(0,0, u)])=\left(\begin{array}{c}
-v^{\top} S u \\
{[X, S] u-a S u} \\
0
\end{array}\right)=\left(\begin{array}{c}
-w^{\top} u \\
w_{+} u \\
0
\end{array}\right)
$$

for all $u \in V_{0}$. This implies that

$$
[X, S]=\left(w_{+} \operatorname{Id}+a S\right) .
$$

Taking the trace yields $w_{+}=0$ and multiplying both sides by $S$ and taking the trace gives

$$
a \operatorname{tr}\left(S^{2}\right)=\operatorname{tr}([X, S] S)=0 .
$$

Since we can chose $a \neq 0$ for both types, this implies that $\operatorname{tr}\left(S^{2}\right)=0$. With $S$ symmetric, we obtain that $S=0$, hence $H^{1}(\mathfrak{a}, V)^{\mathfrak{g}}=\{0\}$ and consequently that $H^{1}(\mathfrak{g}, V)=0$.

Remark 5.8. Similar arguments can be used to determine $H^{1}(\mathfrak{g}, V)$ for the other two types of indecomposable subalgebras of $\mathfrak{s o}(V)_{L}$, those that leave invariant the null vector $\mathrm{e}_{-}$(notations as in Theorem [5.7, for details about these subalgebras see [7]). One of them is of the form $\mathfrak{g}=\mathfrak{g}_{0} \ltimes V_{0}$ and by applying to above arguments to $\mathfrak{b}:=\mathfrak{z} \ltimes V_{0}$ one can show that

$$
H^{1}(\mathfrak{g}, V)=S_{0}\left(V_{0}\right)^{\mathfrak{g}_{0}} \oplus \mathfrak{z}^{*} \oplus\left(V_{0}^{\mathfrak{g}_{0}}\right)^{*},
$$

where $S_{0}\left(V_{0}\right)^{\mathfrak{g}_{0}}$ denotes the trace-free, symmetric matrices that commute with $\mathfrak{g}_{0}$.

A similar statement holds for the remaining fourth type where $\mathfrak{g}=\left(\mathfrak{h}_{f} \oplus \mathfrak{g}_{0}^{\prime}\right) \ltimes T_{0}$, with $0 \neq T_{0} \subsetneq V_{0}$ invariant under $\mathfrak{g}_{0}$ such that $T_{0}^{\perp} \subset \operatorname{ker}\left(\mathfrak{g}_{0}\right)^{\perp}$ and

$$
\mathfrak{h}_{f}=\{(0, Z, f(Z)) \mid Z \in \mathfrak{z}\}, \text { with } f: \mathfrak{z} \rightarrow T_{0}^{\perp} \text { surjective. }
$$

Here one can apply the above strategy to $\mathfrak{b}:=\mathfrak{h}_{f} \ltimes T_{0}$. However, since the result is somewhat technical and we do not need it for what follows, we will not give the details here. 
Finally we study the two types of indecomposable subalgebras of $\mathfrak{s o}(1, n+1)$ that stabilise the null line $L$ but act non trivially on $L$, i.e., the types considered in the previous theorem.

Proposition 5.9. Let $V=\mathbb{R} \cdot \mathrm{e}_{-} \oplus V_{0} \oplus \mathbb{R} \cdot \mathrm{e}_{+}$be the Minkowski space with null vectors $\mathrm{e}_{ \pm}$, and let $\mathfrak{g} \subset \mathfrak{s o}(V)_{L} \subset \mathfrak{s o}(V)$ be an indecomposable subalgebra stabilising a null line $L=\mathbb{R e}_{-}$ but acting non trivially on $L$. Let $\rho \in \mathfrak{g}^{*}$ be defined by the representation of $\mathfrak{g}$ on $V / L^{\perp}$, i.e.,

$$
(a, X, v)[u]=\rho(a, X, v)[u], \quad \text { i.e., } \rho(a, X, v)=-a,
$$

(according to formula (5.2)). Then, every $\varphi \in Z^{1}\left(\mathfrak{g}, V / L^{\perp}\right) \subset \mathfrak{g}^{*}$ is a multiple of $\rho$, or equivalently, $Z^{1}\left(\mathfrak{g}, V / L^{\perp}\right)=d\left(V / L^{\perp}\right)$, i.e., $\left.H^{1}\left(\mathfrak{g}, V / L^{\perp}\right)=\{0\}\right)$.

Proof. First we consider the type $\mathfrak{g}=\left(\mathbb{R} \oplus \mathfrak{g}_{0}\right) \ltimes V_{0}$. Note that we do not exclude the case $V_{0}=0$, for which $\mathfrak{g}=\mathfrak{s o}(1,1)$. For $a \neq 0$, every $\varphi \in Z^{1}\left(\mathfrak{g}, V / L^{\perp}\right)$ satisfies

$$
0=\varphi([(a, 0,0),(0, X, 0)])=-a \varphi(0, X, 0),
$$

for all $X \in \mathfrak{g}_{0}$. Hence $\left.\varphi\right|_{\mathfrak{g}_{0}}=0$. Similarly, we get

$$
a \varphi(0,0, v)=\varphi([(a, 0,0),(0,0, v)])=-a \varphi(0,0, v),
$$

for all $v \in \mathbb{R}^{n}$. Hence $\left.\varphi\right|_{V_{0}}=0$. This implies that $\varphi$ is a multiple of $\rho$.

Now we assume that $\mathfrak{g}=\left(\mathbb{R} \zeta_{0} \oplus \mathfrak{k}\right) \ltimes V_{0}$, where $\mathfrak{k}=\operatorname{ker} f \oplus \mathfrak{g}_{0}^{\prime} \subset \mathfrak{z} \oplus \mathfrak{g}_{0}^{\prime}=\mathfrak{g}_{0}=\operatorname{pr}_{\mathfrak{s o}(n)} \mathfrak{g}$, $f \in \mathfrak{z}^{*}, \zeta_{0}=\left(1, Z_{0}\right)$ and $Z_{0} \in \mathfrak{z}$ is a vector in the centre $\mathfrak{z}$ of $\mathfrak{g}_{0}$ such that $f\left(Z_{0}\right)=1$. In particular, $\operatorname{dim}\left(V_{0}\right) \geqslant 2$. For $X \in \mathfrak{k}$ we obtain

$$
0=\varphi\left(\left[\zeta_{0},(0, X, 0)\right]\right)=-\varphi(0, X, 0),
$$

i.e., $\left.\varphi\right|_{\mathfrak{k}}=0$. Moreover, for all $v \in \mathbb{R}^{n}$ from the cocycle condition we get

$$
\begin{aligned}
-\varphi(0,0, v) & =\varphi\left(\left[\zeta_{0},(0,0, v)\right]\right) \\
& =\varphi\left(0,0,\left(1+Z_{0}\right) v\right)=\varphi(0,0, v)+\varphi\left(0,0, Z_{0} v\right),
\end{aligned}
$$

i.e., that

$$
\varphi\left(0,0, Z_{0} v\right)=-2 \varphi(0,0, v) .
$$

Applying equation (5.8) twice one obtains

$$
\varphi\left(0,0, Z_{0}^{2} v\right)=-2 \varphi\left(0,0, Z_{0} v\right)=4 \varphi(0,0, v) .
$$

Since $Z_{0} \in \mathfrak{s o}(n)$, its square $Z_{0}^{2}$ is diagonalisable with only nonpositive eigenvalues. Hence we get that $\left.\varphi\right|_{V_{0}}=0$. This implies that $\varphi$ is a multiple of $\rho$.

\section{Holonomy of metrics $\widetilde{g}=2 \mathrm{~d} u \mathrm{~d} v+u^{2} g$}

In this section we will use the geometric lifting properties of metrics of the form $\widetilde{g}=$ $2 \mathrm{~d} u \mathrm{~d} v+u^{2} g$ derived in Section 4 and the algebraic results of Section 5 in order study the holonomy of $\widetilde{g}$. For cones over manifolds $(M, g)$ of arbitrary signature but with completely reducible holonomy, Theorem 5.5 has the following consequences.

Corollary 6.1. Let $g$ be a semi-Riemannian metric of signature $(t, s)$ on a manifold $M$ the holonomy algebra $\mathfrak{h o l}(g)$ of which acts completely reducibly. Consider the metric

$$
\widetilde{g}=2 \mathrm{~d} u \mathrm{~d} v+u^{2} g
$$


on $\widetilde{M}=\mathbb{R}^{+} \times \mathbb{R} \times M$ and assume that the holonomy $\tilde{\mathfrak{g}}:=\mathfrak{h} \mathfrak{o r}(\widetilde{g})$ of $\widetilde{g}$ acts indecomposably, i.e. without a proper non-degenerate invariant subspace, and that the translational ideal $T:=\tilde{\mathfrak{g}} \cap V$ is non-degenerate. Then

$$
\mathfrak{h o l}(\widetilde{g})=\mathfrak{h o l}(g) \ltimes V .
$$

Proof. First Proposition 4.2 gives that $\mathfrak{g}=\operatorname{pr}_{\mathfrak{s o}(t, s)}(\tilde{\mathfrak{g}})=\mathfrak{h o l}(g)$. Then Theorem 5.5 applied to $\tilde{\mathfrak{g}}$ shows that $\mathfrak{g} T^{\perp}=0$. If $T^{\perp} \neq\{0\}$, then $g$ admits a non-degenerate parallel vector field which, according to Lemma 4.8, would lift to a non-degenerate parallel vector field for $\widetilde{g}$. This is excluded by the assumption of indecomposability of $\widetilde{g}$.

As an aside, let us record the consequence of Theorem 5.5 for Lorentzian metrics of the form $\tilde{g}=2 \mathrm{~d} u \mathrm{~d} v+u^{2} g$. We have obtained this result in [1, Section 9].

Corollary 6.2. Let $g$ be a Riemannian metric in dimension $n$ and $\widetilde{g}=2 \mathrm{~d} u \mathrm{~d} v+u^{2} g$ a Lorentzian metric. If the holonomy of $\tilde{g}$ acts indecomposably, then

$$
\mathfrak{h o l}(\widetilde{g})=\mathfrak{h o l}(g) \ltimes \mathbb{R}^{n} .
$$

In the main result of this section we deal with metrics $\widetilde{g}$ over Lorentzian metrics $g$.

Theorem 6.3. Let $g$ be a Lorentzian metric on an n-dimensional simply connected manifold $M$ and $\widetilde{g}=2 \mathrm{~d} u \mathrm{~d} v+u^{2} g$ of signature $(2, n)$ on $\mathbb{R}^{+} \times \mathbb{R} \times M$. If the holonomy of $\widetilde{g}$ acts indecomposably, then

$$
\mathfrak{h o l}(\widetilde{g})=\mathfrak{h o l}(g) \ltimes \mathbb{R}^{1, n-1},
$$

or $g$ admits a parallel null vector field and $\widetilde{g}$ admits two linearly independent parallel null vector fields that are orthogonal to each other.

Proof. Set $\tilde{\mathfrak{g}}:=\mathfrak{h o l}(\widetilde{g}), \mathfrak{g}:=\mathfrak{h o l}(g)$ and $V:=\mathbb{R}^{1, n-1}$. Let $T=\tilde{\mathfrak{g}} \cap V$ be the pure translations in $\tilde{\mathfrak{g}}$. We have to show that $T=V$, in which case we have that $\tilde{\mathfrak{g}}=\mathfrak{g} \ltimes V$, or that $\mathfrak{g}$ admits an invariant null vector. Hence we assume from now on that $T \neq V$. By Proposition 4.2 we have that $\tilde{\mathfrak{g}} \subset \mathfrak{g} \ltimes V$ with $\mathfrak{g}=\operatorname{pr}_{\mathfrak{s o}(1, n+1)}(\widetilde{\mathfrak{g}})$ and $T$ is $\mathfrak{g}$ invariant.

Since $\mathfrak{g}$ is a holonomy algebra, we can apply the Wu splitting theorem and obtain $\mathfrak{g}=$ $\mathfrak{g}_{1} \oplus \ldots \oplus \mathfrak{g}_{k}$ and

$$
V=\mathbb{R}^{1, n-1}=V_{0} \oplus^{\perp} V_{1} \oplus^{\perp} V_{2} \oplus^{\perp} \ldots \oplus^{\perp} V_{k},
$$

with $\mathfrak{g}_{i}$ acting trivially on $V_{j}$ for $i \neq j$, all the $V_{i}$ 's are non-degenerate, with $V_{0}$ a trivial representation and $V_{i}$ indecomposable for $i=1, \ldots, k$. Since we assume that $\tilde{\mathfrak{g}}$ acts indecomposably, $\widetilde{g}$ does not admit non-degenerate parallel vector fields. Therefore, Lemma 4.8 implies that $V_{0}=\{0\}$. Hence we can choose the $V_{i}$ in a way that $V_{1}$ is the Minkowski space and indecomposable for $\mathfrak{g}_{1}$ and the remaining $V_{i}$ are Euclidean and irreducible for $\mathfrak{g}_{i}$. Note that for $i=2, \ldots, k$ we have that $\mathfrak{g}_{i} \subset \mathfrak{s o}\left(n_{i}\right)$, where $n_{i}=\operatorname{dim}\left(V_{i}\right)$. Moreover, we can write

$$
\mathfrak{g} \ltimes V=\left(\mathfrak{g}_{1} \oplus \ldots \oplus \mathfrak{g}_{k}\right) \ltimes\left(V_{1} \oplus \ldots \oplus V_{k}\right)=\left(\mathfrak{g}_{1} \ltimes V_{1}\right) \oplus \ldots \oplus\left(\mathfrak{g}_{k} \ltimes V_{k}\right) .
$$

Not only $T$ but also $T_{i}=\tilde{\mathfrak{g}} \cap V_{i}$ is $\mathfrak{g}$-invariant. Hence we have for $i=2, \ldots, k$ that $T_{i}=\{0\}$ or $T_{i}=V_{i}$, and that $T_{1}$ is degenerate, trivial or equal to $V_{1}$. The same holds for $P_{i}=\operatorname{pr}_{V_{i}} T$ containing $T_{i}$.

Since $V_{1}$ is indecomposable but not necessarily irreducible, we have to consider several cases for $T$ :

Case 1: $T$ is indefinite, i.e., of signature $(1, \operatorname{dim}(T)-1)$. In this case we have that $T \cap V_{1}=V_{1}$ and that $T^{\perp}$ is positive definite and hence a direct sum of irreducibles that can 
be arranged such that $T^{\perp}=V_{\ell+1} \oplus \ldots \oplus V_{k}$ with $1 \leqslant \ell \leqslant k-1$ (recall that $T \neq\{0\}$ and that we are working under the assumption $T \neq V$ ). We apply Theorem 5.5 to the following data:

We define $\widetilde{W}:=\operatorname{Re}_{-} \oplus T^{\perp} \oplus \operatorname{Re}_{+}$and a representation $\rho: \tilde{\mathfrak{g}} \rightarrow \mathfrak{s o}(\widetilde{W})_{\mathrm{e}_{-}}$by $\rho(X, v)=$ $\left(\left.X\right|_{T^{\perp}}, \operatorname{pr}_{T^{\perp}}(v)\right)$. Since $T^{\perp}$ is positive definite, it is $T \cap T^{\perp}=\{0\}$, so by its very definition $\rho(\widetilde{\mathfrak{g}})$ satisfies that $\rho(\widetilde{\mathfrak{g}}) \cap T^{\perp}=\{0\}$. On the other hand, $\rho(\widetilde{\mathfrak{g}})$ satisfies the assumptions of Theorem 5.5. Hence, with $\rho(\widetilde{\mathfrak{g}}) \cap T^{\perp}=\{0\}$, the projection of $\rho(\widetilde{\mathfrak{g}})$ onto $\mathfrak{s o}\left(T^{\perp}\right)$ acts trivially on $T^{\perp}$. But this contradicts the fact that $T^{\perp}=V_{\ell+1} \oplus \ldots \oplus V_{k}$, where the $V_{i}$ 's are irreducible for $\operatorname{pr}_{\mathfrak{s o}(1, n-1)}(\widetilde{\mathfrak{g}})$ and hence for $\operatorname{pr}_{\mathfrak{s o}\left(T^{\perp}\right)}(\rho(\tilde{\mathfrak{g}}))$.

Case 2: $T$ is positive definite (including the case $T=0$ ), i.e., $T \cap V_{1}=\{0\}$ in virtue of the indecomposability of the $\mathfrak{g}_{1}$-module $V_{1}$. In this case $T^{\perp}$ is non-degenerate and $V_{1} \subset T^{\perp}$, i.e.,

$$
T^{\perp}=V_{1} \oplus \ldots \oplus V_{\ell} \quad \text { and } \quad T=V_{\ell+1} \oplus \ldots \oplus V_{k} .
$$

Set

$$
\mathfrak{g}_{-}=\mathfrak{g}_{1} \oplus \ldots \oplus \mathfrak{g}_{\ell} \quad \text { and } \quad \mathfrak{g}_{+}=\mathfrak{g}_{\ell+1} \oplus \ldots \oplus \mathfrak{g}_{k}
$$

where $\mathfrak{g}_{+}=\mathfrak{z}_{+}+\mathfrak{g}_{+}^{\prime}$ is reductive with centre $\mathfrak{z}_{+}$and derived algebra $\mathfrak{g}_{+}^{\prime}$, and $\mathfrak{g}_{1}$ is either irreducible or indecomposable but with an invariant null line $L$.

In the case when $\mathfrak{g}_{1}$ acts irreducibly on $V_{1}, \mathfrak{g}$ acts completely reducibly on $V$ and, since $T$ is positive definite, we can apply Corollary 6.1 to get a contradiction to $T \neq V$.

Hence we can assume that $\mathfrak{g}_{1}$ is contained in the stabiliser of the null line $L$, i.e., $\mathfrak{g}_{1} \subset$ $\mathfrak{s o}\left(V_{1}\right)_{L}$. Since $\mathfrak{g}_{+}$acts trivially on $T^{\perp}$ and the $V_{i}$ 's are irreducible for $i=\ell+1, \ldots, k$, and $\mathfrak{g}_{-}$acts trivially on $T$, we have that

$$
V^{\mathfrak{g}-} \cap T^{\perp}=V^{\mathfrak{g}}
$$

As in Proposition 5.1, there is a $\varphi \in Z^{1}\left(\mathfrak{g}, T^{\perp}\right)$, such that $\tilde{\mathfrak{g}}=\mathfrak{h}_{\varphi} \ltimes T$. Then for $X_{ \pm} \in \mathfrak{g}_{ \pm}$ we have

$$
0=\varphi\left(\left[X_{+}, X_{-}\right]\right)=X_{-} \varphi\left(X_{+}\right) .
$$

Hence, using equality (6.1), we obtain $\varphi\left(\mathfrak{g}_{+}\right) \subset V^{\mathfrak{g}_{-}} \cap T^{\perp}=V^{\mathfrak{g}}$. If $\left.\varphi\right|_{\mathfrak{g}_{+}} \neq 0$, we conclude that $V^{\mathfrak{g}}$ is a non-trivial subspace of $T^{\perp}$ and thus $V^{\mathfrak{g}}=L$. Hence, if $\left.\varphi\right|_{\mathfrak{g}_{+}} \neq 0$ there is a non-zero vector in $L$ that is annihilated by $\mathfrak{g}$ and therefore the metric $g$ admits a parallel null vector field.

Hence, for Case 2 we can assume that $\left.\varphi\right|_{\mathfrak{g}_{+}}=0$ and are left with

$$
\varphi: \mathfrak{g}_{-} \longrightarrow T^{\perp}=V_{1} \oplus \ldots \oplus V_{\ell} .
$$

Then for $X_{i} \in \mathfrak{g}_{i}$ and $X_{j} \in \mathfrak{g}_{j}$, with $i, j \in\{1, \ldots, \ell\}$, and $i \neq j$ we have

$$
0=X_{i} \varphi\left(X_{j}\right)-X_{j} \varphi\left(X_{i}\right)
$$

and hence

$$
X_{i} \varphi\left(X_{j}\right)=0 .
$$

Since the $V_{j \geqslant 2}$ are irreducible, this relation for $j=1$ implies that

$$
\left.\varphi\right|_{\mathfrak{g}_{1}} \in Z^{1}\left(\mathfrak{g}_{1}, V_{1}\right)
$$

On the other hand, for $j \geqslant 2$ we have that

$$
\left.\varphi\right|_{\mathfrak{g}_{j}} \in Z^{1}\left(\mathfrak{g}_{j}, L \oplus V_{j}\right),
$$


where $L$ is the $\mathfrak{g}$-invariant null line. If we write $\varphi=\varphi_{1}+\ldots+\varphi_{\ell}$ with $\varphi_{i}: \mathfrak{g}_{-} \rightarrow V_{i}$, then relation (6.2) implies that if there exists $X_{j} \in \mathfrak{g}_{j}$ for some $j \geqslant 2$ such that $\varphi_{1}\left(X_{j}\right) \neq 0$, and thus $\varphi_{1}\left(\mathfrak{g}_{j}\right)=L$, then $\mathfrak{g}_{1}$ and hence $\mathfrak{g}$ acts trivially on $L$. The latter case implies again that the metric $g$ admits a parallel null vector field.

Hence, we have obtained that $g$ admits a parallel null vector field or that $\varphi=\varphi_{1}+\ldots+\varphi_{\ell}$ with $\varphi_{i} \in Z^{1}\left(\mathfrak{g}_{i}, V_{i}\right)$ for $i=1, \ldots \ell$. Since the $V_{i}$ for $i \geqslant 2$ are irreducible, we have that $Z^{1}\left(\mathfrak{g}_{i}, V_{i}\right)=\mathrm{d} V_{i}$, by Lemma 5.6. The case $i=1$ is covered by Theorem 5.7 where we have shown that $H^{1}\left(\mathfrak{g}_{1}, V_{1}\right)=0$ whenever $g$ does not admit a parallel null vector field. Hence, if $g$ does not admit a parallel null vector field we obtain from (1) in Theorem 5.2 that $T^{\perp}$ is degenerate or zero. But this contradicts $T \neq V$ and that $T^{\perp}$ in Case 2 is non-degenerate.

Case 3: $T$ is degenerate, i.e., there is a $\mathfrak{g}$-invariant null line $L=T \cap T^{\perp}$. Our aim is to apply point (2) in Theorem 5.2 and Proposition 5.9, First note that $\mathfrak{g}$ and therefore the indecomposable subalgebra $\mathfrak{g}_{1} \subset \mathfrak{s o}\left(V_{1}\right)$ both leave $T$ and hence the null line $L$ invariant. If $\mathfrak{g}_{1}$ acts trivially on $L$, then $\mathfrak{g}$ acts trivially on $L$ and the metric $g$ admits a parallel null vector field. Therefore we can assume that $\mathfrak{g}_{1}$ does not act trivially on $L$. This means that we can apply Proposition 5.9 to $\mathfrak{g}_{1}$ and $L^{\perp} \cap V_{1}$ to get that

$$
Z^{1}\left(\mathfrak{g}_{1}, V_{1} /\left(L^{\perp} \cap V_{1}\right)\right)=d\left(V_{1} /\left(L^{\perp} \cap V_{1}\right)\right) .
$$

On the other hand, we note that there is a canonical identification

$$
V / L^{\perp} \simeq V_{1} /\left(L^{\perp} \cap V_{1}\right),
$$

which shows that $\mathfrak{g}_{2} \oplus \ldots \oplus \mathfrak{g}_{k}$ acts trivially on $V / L^{\perp}$. Hence,

$$
Z^{1}\left(\mathfrak{g}, V / L^{\perp}\right)=Z^{1}\left(\mathfrak{g}_{1}, V_{1} /\left(L^{\perp} \cap V_{1}\right)\right)=d\left(V / L^{\perp}\right) .
$$

Since we have assumed that $\mathfrak{g}$ does not act trivially on $L,(2)$ in Theorem 5.2 implies that, up to conjugation, $\tilde{\mathfrak{g}}$ leaves invariant a null line $L$. This means that $(\widetilde{M}, \widetilde{g})$ admits a recurrent null vector field in the span of $\partial_{v}$ and $L$ (even a recurrent section in $L$ ). But in this situation, Proposition 4.10 ensures the existence of a parallel null vector field on $(M, g)$.

\section{Cones with parallel null 2-Planes}

In this section we consider the base manifolds $(M, g)$ of cones that admit a parallel distribution of totally null 2-planes. Our main result is the description of the most general local form of the metric $g$. To exclude trivial cases we assume $\operatorname{dim} M>1$.

7.1. The induced structure on the base. If $(\widehat{M}, \widehat{g})$ is a semi-Riemannian manifold and $\widehat{\mathbf{P}}$ a parallel totally null 2-plane bundle, then locally there are two null vector fields $\chi$ and $\zeta$ that are orthogonal to each other and such that

$$
\begin{aligned}
& \hat{\nabla} \chi=\alpha \otimes \chi+\mu \otimes \zeta, \\
& \hat{\nabla} \zeta=\beta \otimes \chi+\nu \otimes \zeta,
\end{aligned}
$$

for 1 -forms $\alpha, \beta, \mu$ and $\nu$.

If $(\widehat{M}, \widehat{g})$ is a timelike cone with a parallel null 2-plane bundle $\widehat{\mathbf{P}}$, we can intersect $\widehat{\mathbf{P}}$ with $\xi^{\perp}$, where $\xi$ is the Euler vector field. A subset of $\widehat{M}=\mathbb{R}^{>0} \times M$ will be called conical if it is of the form $\widehat{M}_{0}=\mathbb{R}^{>0} \times M_{0}$ for some subset $M_{0} \subset M$. 
Lemma 7.1. On a conical open dense subset in $\widehat{M}$ the intersection $\widehat{\mathbf{P}} \cap \xi^{\perp}$ is a null-line bundle $\mathbf{L}$ invariant under the flow of $\xi$. In particular, $\mathbf{L}$ admits local sections, defined on conical open sets, invariant under the flow of $\xi$ and descends to a null line distribution on an open dense subset of $M$.

Proof. For this and the following proofs, we note that

$$
\left[\xi, \Gamma\left(\xi^{\perp}\right)\right] \subset \Gamma\left(\xi^{\perp}\right) \text { and } \quad[\xi, \Gamma(\widehat{\mathbf{P}})] \subset \Gamma(\widehat{\mathbf{P}}) .
$$

This implies that the dimension of the fibres of $\widehat{\mathbf{P}} \cap \xi^{\perp}$ is constant on the integral curves of $\xi$. At each point $p \in \widehat{M},\left.\xi^{\perp}\right|_{p}$ is a hyperplane and $\left.\widehat{\mathbf{P}}\right|_{p}$ a 2-plane in $T_{p} \widehat{M}$. Hence their intersection has dimension one or two. Now let us assume that, over an open set $U \subset \widehat{M}$, $\widehat{\mathbf{P}} \cap \xi^{\perp}$ is of rank 2, i.e. that $\widehat{\mathbf{P}} \subset \xi^{\perp}$. Hence $\widehat{\mathbf{P}} \cap \xi^{\perp}$ a distribution of 2-planes spanned by vector fields $V_{1}$ and $V_{2}$ on $U$ that are tangential to $M$. Then formulae (2.2) and (7.1) give us

$$
T M \ni \hat{\nabla}_{X} V_{i}=\nabla_{X} V_{i}+g\left(X, V_{i}\right) \xi
$$

for all $X \in T M$. Hence, on $U$ it is $g\left(X, V_{i}\right)=0$ for all $X \in T M$ which is impossible. Consequently, the conical open set over which the fibres of $\widehat{\mathbf{P}} \cap \xi^{\perp}$ are one-dimensional is dense and $\widehat{\mathbf{P}} \cap \xi^{\perp}$ restricts to a line bundle $\mathbf{L}$ over that set.

Now we project $\widehat{\mathbf{P}}$ to $\xi^{\perp}$.

Lemma 7.2. The projection $\operatorname{pr}_{\xi^{\perp}}(\widehat{\mathbf{P}}) \subset \xi^{\perp}$ is an involutive 2-plane distribution $\mathbf{P}$ on $\widehat{M}$ and descends to an involutive 2-plane distribution on $M_{0}$.

Proof. First note that the fibres of $\operatorname{pr}_{\xi^{\perp}}(\widehat{\mathbf{P}})$ have dimension 2 because $\widehat{\mathbf{P}} \cap \mathbb{R} \cdot \xi=\{0\}$. Hence, $\mathbf{P}:=\operatorname{pr}_{\xi^{\perp}}(\widehat{\mathbf{P}}) \subset \xi^{\perp}$ is a 2-plane distribution.

Clearly the projection of a vector field $V$ on $\widehat{M}$ to $\xi^{\perp}$ is given as

$$
\operatorname{pr}_{\xi^{\perp}}(V)=V+r^{-2} \widehat{g}(V, \xi) \xi .
$$

By a calculation using $\hat{\nabla} \xi=$ Id we obtain for all $V_{1}, V_{2} \in \mathfrak{X}(\widehat{M})$ :

$$
\left[\operatorname{pr}_{\xi^{\perp}}\left(V_{1}\right), \operatorname{pr}_{\xi^{\perp}}\left(V_{2}\right)\right]=\operatorname{pr}_{\xi^{\perp}}\left(\left[V_{1}, V_{2}\right]+r^{-2} \widehat{g}\left(V_{2}, \xi\right)\left[V_{1}, \xi\right]-r^{-2} \widehat{g}\left(V_{1}, \xi\right)\left[V_{2}, \xi\right]\right) .
$$

Since the distribution $\widehat{\mathbf{P}}$ is invariant under $\xi$, parallel and hence involutive, the right-hand side is a section of $\mathbf{P}$ for all sections $V_{1}, V_{2}$ of $\widehat{\mathbf{P}}$. This proves the involutivity of $\mathbf{P}$. The distribution $\mathbf{P}$ descends to $M$ due to the invariance under $\xi$.

Moreover we obtain:

Lemma 7.3. There exist local sections $V$ of $\mathbf{L}$ and $Z$ of $\mathbf{P}$, defined on a conical open set, such that $V$ and

$$
\zeta=\xi+Z
$$

locally span $\widehat{\mathbf{P}}$ and satisfy

$$
[\xi, V]=0 \quad \text { and } \quad[\xi, Z]=0 .
$$

The vector fields $V$ and $Z$ descend to local vector fields on $M$. 
Proof. We have already seen that there exists a non-vanishing section $V$ of $\mathbf{L}$ over a conical open set such that $[\xi, V]=0$. In the following we always work locally over conical open sets. Every section of $\widehat{\mathbf{P}}$ that is nowhere a multiple of $V$ is of the form $f \xi+Z$ for $Z$ a (possibly vanishing) local section of $\mathbf{P}$ and $f$ a non-vanishing local function on $\widehat{M}$. Hence, by multiplying with $1 / f$ we can assume that we have a section

$$
\hat{\zeta}=\xi+\hat{Z}
$$

of $\widehat{\mathbf{P}}$. We will now use the freedom to add multiples of $V$ to $\hat{Z}$ without leaving $\widehat{\mathbf{P}}$, in order to find a $Z=\hat{Z}+\varphi V$ for which we have $[\xi, Z]=0$. Indeed, writing

$$
\nabla_{\xi} \hat{\zeta}=f V+h \hat{\zeta}
$$

with functions $f$ and $h$, we compute

$$
[\xi, \hat{Z}]=[\xi, \hat{\zeta}]=f V+(h-1) \hat{\zeta} .
$$

Since $[\xi, \hat{Z}]$ belongs to $\xi^{\perp}$, we must have that $h \equiv 1$ and

$$
[\xi, \hat{Z}]=f V .
$$

Now if we fix a solution $\varphi$ of

$$
\mathrm{d} \varphi(\xi)+f=0
$$

and set $Z=\hat{Z}+\varphi V$ we get

$$
[\xi, Z]=0 .
$$

Clearly, since $V$ is a section of $\hat{\mathbf{P}}$, the vector field

$$
\zeta:=\xi+Z=\hat{\zeta}+\varphi V
$$

is also a section in $\widehat{\mathbf{P}}$ that is still linearly independent of $V$ and therefore $Z$ is a section of $\mathbf{P}$ that locally descends to $M$.

Theorem 7.4. Let $(\widehat{M}, \widehat{g})$ be a timelike cone over a semi-Riemannian manifold $(M, g)$. If the cone admits a parallel distribution of totally null 2-planes field, then the base $(M, g)$ admits locally two vector fields $V$ and $Z$ such that

$$
g(V, V)=0, \quad g(Z, Z)=1, \quad g(V, Z)=0,
$$

and

$$
\begin{aligned}
& \nabla_{X} V=\alpha(X) V+g(X, V) Z, \\
& \nabla_{X} Z=-X+\beta(X) V+g(X, Z) Z,
\end{aligned}
$$

for all $X \in T M$, with 1 -forms $\alpha$ and $\beta$ on $M$.

Conversely, each pair of vector fields $V$ and $Z$ on $M$ satisfying relations (7.2), (7.3) and 77.4) defines a parallel distribution of totally null 2-planes on the cone.

Proof. First assume that the cone admits a parallel totally null 2-plane $\widehat{\mathbf{P}}$ which is spanned by $V$ and $\zeta=\xi+Z$ as in Lemma [7.3. Equations (7.2) are implied by $\hat{\mathbf{P}}$ being totally null. Moreover, equations (7.1) with $\chi=V$ and $X \in T M$ become

$$
\begin{aligned}
\hat{\nabla}_{X} V=\nabla_{X} V+g(X, V) \xi & =\alpha(X) V+\mu(X)(\xi+Z), \\
\hat{\nabla}_{X} \zeta=X+\nabla_{X} Z+g(X, Z) \xi & =\beta(X) V+\nu(X)(\xi+Z),
\end{aligned}
$$


and imply

$$
\begin{aligned}
& \mu(X)=g(X, V), \\
& \nu(X)=g(X, Z),
\end{aligned}
$$

as well as equations (7.3) and (7.4), but still with $r$-dependent 1 -forms $\alpha$ and $\beta$. Hence, it remains to show that $\alpha$ and $\beta$, when restricted to $\xi^{\perp}$, are invariant under the flow of $\xi$ and therefore descend to 1 -forms on $M$, i.e., that

$$
\left.\mathcal{L}_{\xi} \alpha\right|_{\xi^{\perp}}=\left.\mathcal{L}_{\xi} \beta\right|_{\xi^{\perp}}=0
$$

But from

$$
\begin{aligned}
0 & =\widehat{R}(\xi, X) V \\
& =\left(\mathcal{L}_{\xi} \alpha\right)(X) V+\alpha(X) V+g(X, V)(\xi+Z)-\left(\nabla_{X} V+g(X, V) \xi\right) \\
& =\left(\mathcal{L}_{\xi} \alpha\right)(X) V
\end{aligned}
$$

because of equation (7.5). This proves that $\left.\mathcal{L}_{\xi} \alpha\right|_{\xi^{\perp}}=0$. Analogously we get

$$
0=\widehat{R}(\xi, X) \zeta=\left(\mathcal{L}_{\xi} \beta\right)(X) V
$$

and again $\left.\mathcal{L}_{\xi} \beta\right|_{\xi^{\perp}}=0$.

Conversely, if we start with a manifold $(M, g)$ and vector fields satisfying conditions (7.2), (17.3) and (7.4), a straightforward computations shows that the cone admits a parallel null plane spanned by $V$ and $\xi+Z$.

Corollary 7.5. If the cone (2.1) admits a distribution of parallel totally null 2-planes, then the base $(M, g)$ admits locally a geodesic, shearfree null vector field $V$.

Proof. Since $V$ is null, equation (7.3) implies that $V$ is geodesic. Recall that a geodesic null vector field is called shearfree if

$$
\mathcal{L}_{V} g=\lambda g+\theta \cdot V^{b}
$$

with a function $\lambda$ and a 1 -form $\theta$ and where the dot stands for the symmetric product. From (7.3) and the formula

$$
\mathcal{L}_{X} g=2\left(\nabla X^{b}\right)^{\mathrm{sym}},
$$

where 'sym' denotes the projection onto the symmetric part, we compute

$$
\mathcal{L}_{V} g=2\left(\alpha+Z^{b}\right) \cdot V^{b},
$$

i.e., the shear free condition is satisfied with $\lambda=0$.

Remark 7.6. We can change the basis of $\operatorname{span}(V, Z)$ to $V^{\prime}, Z^{\prime}$ such that $V^{\prime}$ is still null and orthogonal to $Z^{\prime}$ and such that $Z^{\prime}$ is a unit vector field,

$$
(V, Z) \longmapsto\left(V^{\prime}=\mathrm{e}^{f} V, Z^{\prime}=Z+h V\right) .
$$

Then the 1-forms $\alpha$ and $\beta$ transform as

$$
\begin{aligned}
& \alpha \quad \alpha^{\prime}=\alpha+\mathrm{d} f-h V^{b}, \\
& \beta \quad \beta^{\prime}=e^{-f}\left(\beta+h \alpha+\mathrm{d} h-h Z^{b}-h^{2} V^{b}\right) .
\end{aligned}
$$


7.2. Consequences of the fundamental equations. Let $(M, g)$ be a semi-Riemannian manifold endowed with two pointwise linearly independent vector fields $V, Z$ which satisfy (7.2), (7.3) and (77.4).

Proposition 7.7. The fundamental equations (7.2 7.3 and 7.4 imply

$$
\begin{aligned}
d V^{b} & =\left(\alpha-Z^{b}\right) \wedge V^{b}, \\
d Z^{b} & =\beta \wedge V^{b}, \\
{[Z, V] } & =(\alpha(Z)-\beta(V)+1) V, \\
\mathcal{L}_{V} g & =2\left(\alpha+Z^{b}\right) V^{b} \\
\mathcal{L}_{Z} g & =-2 g+2\left(Z^{b}\right)^{2}+2 \beta V^{b},
\end{aligned}
$$

where we are using the symmetric product of 1-forms in the last two formulas.

Proof. Since $\nabla$ is torsion-free, the differential of any 1 -form $\varphi$ is given by

$$
\mathrm{d} \varphi(X, Y)=\left(\nabla_{X} \varphi\right) Y-\left(\nabla_{Y} \varphi\right) X, \quad X, Y \in \mathfrak{X}(M) .
$$

Now (7.8) and (7.9) follow immediately from (7.3) and (7.4). Using again that $\nabla$ is torsionfree, the fundamental equations easily imply (7.10). Similarly, the last two formulas follow from (7.7).

Corollary 7.8. We have

$$
\begin{aligned}
\mathcal{L}_{V} V^{b} & =\alpha(V) V^{b}, \\
\mathcal{L}_{Z} V^{b} & =(\alpha(Z)-1) V^{b}, \\
\mathcal{L}_{V} Z^{b} & =\beta(V) V^{b} .
\end{aligned}
$$

The vector fields $Z$ and $V$ commute if and only if

$$
\beta(V)=\alpha(Z)+1 \text {. }
$$

Proof. The first three formulas are obtained from Cartan's formula for the Lie derivative to the equations (7.8) and (7.9). Alternatively one can use (7.10), (7.11) and (7.12). The last assertion follows from equation (7.10).

Corollary 7.9. By multiplying $V$ with a function we can locally assume that

$$
\mathrm{d} V^{b}=0,
$$

that is

$$
\alpha=Z^{b}+f_{\alpha} V^{b}
$$

for some function $f_{\alpha}$. The latter equation implies

$$
\alpha(Z)=1, \quad \alpha(V)=0, \quad \mathcal{L}_{V} V^{b}=0, \quad \mathcal{L}_{Z} V^{b}=0 .
$$

By adding a functional multiple of $V$ to $Z$ we can further locally assume that

$$
\beta(V)=2,
$$

which implies $\mathcal{L}_{V} Z^{b}=2 V^{b}$ and is equivalent to $[Z, V]=0$. 
Proof. By equation (7.8) and the Frobenius theorem, the hyperplane distribution $V^{\perp}$ is integrable, which locally implies that a functional multiple of $V^{\mathfrak{b}}$ is closed. The equations and the second statement follow from the transformation formulae for $\alpha$ and $\beta$ in Remark 7.6 and Corollary 7.8 ,

Corollary 7.10. With the normalisation that $d V^{b}=0$, the leaves of the integrable distribution $V^{\perp}$ are totally geodesic and the vector field $V$ preserves the tensor field $\left.g\right|_{V^{\perp} \times V^{\perp}}$.

Proof. For $X, Y \in V^{\perp}$ we have

$$
g\left(\nabla_{X} Y, V\right)=-g\left(Y, \nabla_{X} V\right)
$$

and because of $d V^{b}=0$,

$$
g\left(Y, \nabla_{X} V\right)=\frac{1}{2}\left(\mathcal{L}_{V} g\right)(X, Y)
$$

Using equation (7.11) for $X, Y \in V^{\perp}$ we get $\left.\mathcal{L}_{V} g\right)(X, Y)=0$ and hence $g\left(\nabla_{X} Y, V\right)=0$, which means that the leaves of $V^{\perp}$ are totally geodesic.

7.3. The local form of the metric on the base. In the following we will assume all of the above equations. By (7.17), locally, there exists a function $u$ such that $d u=V^{b}$. The function $u$ is constant on each leaf $L$ of the distribution $V^{\perp}$. Locally, we can decompose $M$ as $M=L \times \mathbb{R}$, such that $u$ corresponds to the coordinate on the $\mathbb{R}$-factor and the leafs of $V^{\perp}$ are the hypersurfaces $L_{u}=L \times\{u\}$. Since the vector fields $V$ and $Z$ commute and are tangent to $V^{\perp}$, we can further decompose each leaf of $V^{\perp}$ locally as $L_{u} \cong L=M_{0} \times \mathbb{R} \times \mathbb{R}$, such that $V=\partial_{t}, Z=\partial_{s}$ are the coordinate vector fields tangent to the first and second $\mathbb{R}$-factor, respectively.

Let us denote by $\mathbf{P}$ the integrable distribution spanned by $V$ and $Z$. Notice that by (7.9) the distribution $\mathbf{P}^{\perp}=Z^{\perp} \cap V^{\perp}$ is also integrable, in virtue of the Frobenius theorem. So we can assume that the level sets of $s$ are tangent to $\mathbf{P}^{\perp}$. Finally, the decomposition $M=L \times \mathbb{R}$ can be chosen such that the decomposition $L_{u}=M_{0} \times \mathbb{R} \times \mathbb{R}$ is independent of $u$, that is the vector field $\partial_{u}$ commutes with $V, Z$ and with the canonical lift of vector fields of $M_{0}$.

Theorem 7.11. Let $(M, g)$ be a semi-Riemannian manifold such that the cone $(\widehat{M}, \widehat{g})$ admits a parallel totally null distribution of 2-planes. In terms of the above local decomposition $M=M_{0} \times \mathbb{R}^{3}$ we have

$$
g=\mathrm{d} s^{2}+e^{-2 s} g_{0}(u)+2 \mathrm{~d} u \eta,
$$

for some 1-form $\eta$ on $M$ such that $\eta\left(\partial_{t}\right)$ is nowhere vanishing and a family of metrics $g_{0}(u)$ on $M_{0}$ depending on $u$.

Proof. The restriction of the metric to a leaf $N=M_{0} \times \mathbb{R} \times\{(s, u)\}$ of $\mathbf{P}^{\perp}$ is degenerate with kernel $V=\partial_{t} \in \mathbf{P}^{\perp}$ and invariant under the flow of $V$, see (17.11). Since $M_{0}$ is transversal to $V$, we see that $\left.g\right|_{N}=g_{0}(u, s)$ for some family of metrics on $M_{0}$ depending on $u$ and $s$. The flow of $Z=\partial_{s}$ is a 1-parameter family of homotheties of weight -2 , see (7.12). This shows that $g_{0}(u, s)=e^{-2 s} g_{0}(u)$ for some 1-parameter family of metrics $g_{0}(u)$. It follows that on the leafs $L_{u}=M_{0} \times \mathbb{R} \times \mathbb{R} \times\{u\}$ of $V^{\perp}$ the metric is of the form $\mathrm{d} s^{2}+e^{-2 s} g_{0}(u)$. Finally, on $M$ we obtain the general form (7.18) with $\eta\left(\partial_{t}\right) \neq 0$, in view of the non-degeneracy of $g$. 
It remains to determine the necessary and sufficient conditions for the data $g_{0}(u)$ and $\eta$ ensuring that the cone over $(M, g)$ as in (17.18) admits a parallel totally null distribution of 2-planes. Let $M_{0}$ be a manifold and let us denote the standard coordinates on $\mathbb{R}^{3}$ by $(t, s, u)$.

Theorem 7.12. For any 1-form $\eta$ on $M:=M_{0} \times \mathbb{R}^{3}$ such that $\eta_{t}:=\eta\left(\partial_{t}\right) \neq 0$ and any family of semi-Riemannian metrics $g_{0}(u)$ on $M_{0}$ the tensor field

$$
g=\mathrm{d} s^{2}+e^{-2 s} g_{0}(u)+2 d u \eta,
$$

cf. 7.18), is a semi-Riemannian metric on $M$ such that the vector fields $V=\partial_{t}$ and $Z=\partial_{s}$ satisfy (7.2). The covariant derivatives of $V$ and $Z$ are given by (7.3) and (7.4) for some 1 -forms $\alpha=Z^{b}+f_{\alpha} V^{b}$ and $\beta$ such that $f_{\alpha}$ is a function on $M$ and $\beta(V)=2$, if and only if the coefficients of $\eta$ solve the following system of first order partial differential equations:

$$
\partial_{t} \eta_{t}=\partial_{s} \eta_{t}=X \eta_{t}=\partial_{t} \eta(X)=0, \quad \partial_{t} \eta_{s}=2 \eta_{t}, \quad \partial_{s} \eta(X)-X \eta_{s}=-2 \eta(X)
$$

for all $X \in \mathfrak{X}\left(M_{0}\right)$. Then $\alpha$ and $\beta$ are determined by

$$
\begin{aligned}
& f_{\alpha}=\frac{1}{\eta_{t}^{2}} \partial_{t} \eta_{u}-\frac{2}{\eta_{t}} \eta_{s}, \quad \beta(Z)=\frac{1}{\eta_{t}} \partial_{s} \eta_{s}, \quad \beta(X)=\frac{1}{2 \eta_{t}}\left(X \eta_{s}+\partial_{s} \eta(X)+2 \eta(X)\right), \\
& \beta\left(\partial_{u}\right)=\frac{1}{\eta_{t}}\left(\partial_{s} \eta_{u}-\eta_{s}^{2}+2 \eta_{u}\right) .
\end{aligned}
$$

Proof. We denote by $X$ the canonical lift of a vector field on $M_{0}$. Then $X, V, Z$ and $\partial_{u}$ commute and using the Koszul formula we obtain

$$
\begin{aligned}
& g\left(\nabla_{V} V, X\right)=g\left(\nabla_{V} V, V\right)=g\left(\nabla_{V} V, Z\right)=0, \quad g\left(\nabla_{V} V, \partial_{u}\right)=\partial_{t} \eta_{t}, \\
& g\left(\nabla_{Z} V, X\right)=g\left(\nabla_{Z} V, V\right)=g\left(\nabla_{Z} V, Z\right)=0, \quad 2 g\left(\nabla_{Z} V, \partial_{u}\right)=\partial_{s} \eta_{t}+\partial_{t} \eta_{s}, \\
& g\left(\nabla_{X} V, X\right)=g\left(\nabla_{X} V, V\right)=g\left(\nabla_{X} V, Z\right)=0, \quad 2 g\left(\nabla_{X} V, \partial_{u}\right)=X \eta_{t}+\partial_{t} \eta(X), \\
& 2 g\left(\nabla_{\partial_{u}} V, X\right)=\partial_{t} \eta(X)-X \eta_{t}, \quad g\left(\nabla_{\partial_{u}} V, V\right)=0, \quad 2 g\left(\nabla_{\partial_{u}} V, Z\right)=\partial_{t} \eta_{s}-\partial_{s} \eta_{t}, \\
& g\left(\nabla_{\partial_{u}} V, \partial_{u}\right)=\partial_{t} \eta_{u}, \\
& g\left(\nabla_{V} Z, X\right)=g\left(\nabla_{V} Z, V\right)=g\left(\nabla_{V} Z, Z\right)=0, \quad 2 g\left(\nabla_{V} Z, \partial_{u}\right)=\partial_{t} \eta_{s}+\partial_{s} \eta_{t}, \\
& g\left(\nabla_{Z} Z, X\right)=g\left(\nabla_{Z} Z, V\right)=g\left(\nabla_{Z} Z, Z\right)=0, \quad g\left(\nabla_{Z} Z, \partial_{u}\right)=\partial_{s} \eta_{s}, \\
& g\left(\nabla_{X} Z, X\right)=-g(X, X), \quad g\left(\nabla_{X} Z, V\right)=g\left(\nabla_{X} Z, Z\right)=0, \\
& 2 g\left(\nabla_{X} Z, \partial_{u}\right)=X \eta_{s}+\partial_{s} \eta(X), \\
& 2 g\left(\nabla_{\partial_{u}} Z, X\right)=\partial_{s} \eta(X)-X \eta_{s}, \quad 2 g\left(\nabla_{\partial_{u}} Z, V\right)=\partial_{s} \eta_{t}-\partial_{t} \eta_{s}, \quad g\left(\nabla_{\partial_{u}} Z, Z\right)=0, \\
& g\left(\nabla_{\partial_{u}} Z, \partial_{u}\right)=\partial_{s} \eta_{u} .
\end{aligned}
$$

Comparing with (7.3), (7.4) we obtain the above formulas for $\alpha$ and $\beta$ and the following system for $\eta$ :

$$
\begin{aligned}
& \partial_{t} \eta_{t}=0, \quad \partial_{s} \eta_{t}+\partial_{t} \eta_{s}=2 \eta_{t}, \quad X \eta_{t}+\partial_{t} \eta(X)=0, \quad \partial_{t} \eta(X)-X \eta_{t}=0 \\
& \partial_{t} \eta_{s}-\partial_{s} \eta_{t}=2 \eta_{t} \\
& \partial_{s} \eta(X)-X \eta_{s}=-2 \eta(X)
\end{aligned}
$$

for all $X \in \mathfrak{X}\left(M_{0}\right)$. This system can be brought to the form (7.19). 
For convenience we denote a system of local coordinates on $M_{0}$ by $\left(x^{i}\right)_{i=1, \ldots, n_{0}}$ and denote by $x$ the corresponding coordinate vector, where $n_{0}=\operatorname{dim} M_{0}$. The general solution of (7.19) is obtained as follows.

Proposition 7.13. Let $f_{1}=f_{1}(u)$ be an arbitrary nowhere vanishing smooth function on the real line equipped with the coordinate $u$ and $f_{2}=f_{2}(x, s, u)$ an arbitrary smooth function on $M$ which does not depend on $t$. Let $h_{i}=h_{i}(x, s, u)$ be a (t-independent) solution of the ordinary differential equation

$$
\partial_{s} h_{i}+2 h_{i}=\partial_{i} f_{2}
$$

for all $i=1, \ldots, n_{0}$, where $\partial_{i}=\partial / \partial x^{i}$. Then

$$
\eta_{t}:=f_{1}(u), \quad \eta_{s}:=2 t f_{1}(u)+f_{2}(x, s, u), \quad \eta\left(\partial_{i}\right):=h_{i}(x, s, u)
$$

solves 7.19) and every solution is of this form.

Remark 7.14. Finally we return to the Lorentzian metrics that occurred in Theorem 1.3 and arose from the case where the cone $(\widehat{M}, \widehat{g})$ admits a parallel null line: in this case the cone metric $\hat{g}$ was isometric to the metric $\widetilde{g}=2 \mathrm{~d} u \mathrm{~d} v+u^{2} g_{0}$ with a Lorentzian metric $g_{0}$ and $g$ was isometric to $g=\mathrm{d} s^{2}+\mathrm{e}^{2 s} g_{0}$. Then Theorem 1.3 stated that if the holonomy of the cone is not equal to $\mathfrak{h o l}\left(g_{0}\right) \ltimes \mathbb{R}^{1, n-1}$, then $g_{0}$ admits a parallel null vector field. It is well known (see for example [27, 16]) that locally $g_{0}$ is of the form $g_{0}=2 \mathrm{~d} x \mathrm{~d} z+h(z)$, where $h(z)$ is a $z$-dependent family of Riemannian metrics. Hence, $g$ is of the form

$$
g=d s^{2}+\mathrm{e}^{2 s} h(z)+2 \mathrm{e}^{2 s} \mathrm{~d} x \mathrm{~d} z .
$$

This corresponds to the local form in Theorem [7.12, where $x$ corresponds to $t$ and $2 \mathrm{e}^{2 s} \mathrm{~d} x$ to $\eta, z$ to $u$ and $h(z)$ to $g_{0}(u)$.

\section{REFERENCES}

[1] D. Alekseevsky, V. Cortés, A. Galaev, and T. Leistner. Cones over pseudo-Riemannian manifolds and their holonomy. J. Reine Angew. Math., 635:23-69, 2009.

[2] D. V. Alekseevsky and V. Cortés. Classification of pseudo-Riemannian symmetric spaces of quaternionic Kähler type. In Lie groups and invariant theory, volume 213 of Amer. Math. Soc. Transl. Ser. 2, pages 33-62. Amer. Math. Soc., Providence, RI, 2005.

[3] S. Armstrong. Projective holonomy. II. Cones and complete classifications. Ann. Global Anal. Geom., 33(2):137-160, 2008.

[4] C. Bär. Real Killing spinors and holonomy. Commun. Math. Phys., 154(3):509-521, 1993.

[5] H. Baum. Complete Riemannian manifolds with imaginary Killing spinors. Ann. Global Anal. Geom., 7(3):205-226, 1989.

[6] H. Baum. Odd-dimensional Riemannian manifolds with imaginary Killing spinors. Ann. Global Anal. Geom., 7(2):141-153, 1989.

[7] L. Bérard-Bergery and A. Ikemakhen. On the holonomy of Lorentzian manifolds. In Differential Geometry: Geometry in Mathematical Physics and Related Topics (Los Angeles, CA, 1990), volume 54 of Proc. Sympos. Pure Math., pages 27-40. Amer. Math. Soc., Providence, RI, 1993.

[8] M. Berger. Sur les groupes d'holonomie homogène des variétés à connexion affine et des variétés riemanniennes. Bull. Soc. Math. France, 83:279-330, 1955.

[9] V. Cortés, T. Leistner, L. Schäfer, and F. Schulte-Hengesbach. Half-flat structures and special holonomy. Proc. Lond. Math. Soc., 102(3):113-158, 2010.

[10] G. de Rham. Sur la reductibilité d'un espace de Riemann. Comment. Math. Helv., 26:328-344, 1952.

[11] A. J. Di Scala and C. Olmos. The geometry of homogeneous submanifolds of hyperbolic space. Math. Z., 237(1):199-209, 2001.

[12] A. Fedorova and V. S. Matveev. Degree of mobility for metrics of Lorentzian signature and parallel (0, 2)-tensor fields on cone manifolds. Proc. Lond. Math. Soc. (3), 108(5):1277-1312, 2014. 
[13] C. Fefferman and C. R. Graham. Conformal invariants. Astérisque, (Numero Hors Serie):95-116, 1985. The mathematical heritage of Élie Cartan (Lyon, 1984).

[14] C. Fefferman and C. R. Graham. The ambient metric, volume 178 of Annals of Mathematics Studies. Princeton University Press, Princeton, NJ, 2012.

[15] A. S. Galaev. Holonomy groups and special geometric structures of pseudo-Kählerian manifolds of index 2. PhD thesis, Humboldt University Berlin, Dec. 2006. arXiv:math/0612392.

[16] A. S. Galaev and T. Leistner. On the local structure of Lorentzian Einstein manifolds with parallel distribution of null lines. Classical Quantum Gravity, 27(22):5003, 2010.

[17] S. Gallot. Équations différentielles caractéristiques de la sphère. Ann. Sci. École Norm. Sup. (4), 12(2):235-267, 1979.

[18] G. W. Gibbons and P. Rychenkova. Cones, tri-Sasakian structures and superconformal invariance. Phys. Lett. B, 443(1-4):138-142, 1998.

[19] G. Hochschild and J.-P. Serre. Cohomology of Lie algebras. Ann. of Math. (2), 57:591-603, 1953.

[20] I. Kath. Killing Spinors on Pseudo-Riemannian Manifolds. 1999. Habilitationsschrift, HumboldtUniversität Berlin.

[21] T. Leistner. Conformal holonomy of C-spaces, Ricci-flat, and Lorentzian manifolds. Differential Geom. Appl., 24(5):458-478, 2006.

[22] V. S. Matveev. Gallot-Tanno theorem for pseudo-Riemannian metrics and a proof that decomposable cones over closed complete pseudo-Riemannian manifolds do not exist. Differential Geom. Appl., 28(2):236-240, 2010.

[23] V. S. Matveev and P. Mounoud. Gallot-Tanno theorem for closed incomplete pseudo-Riemannian manifolds and applications. Ann. Global Anal. Geom., 38(3):259-271, 2010.

[24] P. Mounoud. Parallel and symmetric 2-tensor fields on pseudo-Riemannian cones. Comm. Anal. Geom., 20(1):203-233, 2012.

[25] B. O'Neill. Semi-Riemannian Geometry. Academic Press, 1983.

[26] L. Schäfer and F. Schulte-Hengesbach. Nearly pseudo-Kähler and nearly para-Kähler six-manifolds. In Handbook of pseudo-Riemannian geometry and supersymmetry, volume 16 of IRMA Lect. Math. Theor. Phys., pages 425-453. Eur. Math. Soc., Zürich, 2010.

[27] R. Schimming. Riemannsche Räume mit ebenfrontiger und mit ebener Symmetrie. Mathematische Nachrichten, 59:128-162, 1974.

[28] M. Solleveld. Lie algebra cohomology and Macdonalds conjectures. Master's thesis, University of Amsterdam, Korteweg-De Vries Institute for Mathematics, 2002. https://www.math.ru.nl/ solleveld/scrip.pdf.

[29] T. Y. Thomas. Announcement of a projective theory of a affinely connected manifolds. Proc. Nat. Acad. Sci., 11:588-589, 1925.

[30] M. Y. Wang. Parallel spinors and parallel forms. Ann. Global Anal. Geom., 7(1):59-68, 1989.

[31] H. Wu. On the de Rham decomposition theorem. Illinois J. Math., 8:291-311, 1964.

Institute for Information Transmission Problems, B. Karetnuj Per., 19, 127951, Moscow, Russia and University of Hradec Králové, Faculty of Science, Rokitanského 62, 50003 Hradec Králové, Czech Republic

Email address: dalekseevsky@iitp.ru

Department Mathematik, Universität Hamburg, Bundesstrasse 55, D-20146 Hamburg, GerMANY

Email address: vicente.cortes@uni-hamburg.de

School of Mathematical Sciences, University of Adelaide, SA 5005, Australia

Email address: thomas.leistner@adelaide.edu.au 